\title{
Mena/VASP and all-Spectrin complexes regulate cytoplasmic actin networks in cardiomyocytes and protect from conduction abnormalities and dilated cardiomyopathy
}

Peter M Benz ${ }^{1,2^{*}}$, Carla J Merkel ${ }^{1,3}$, Kristin Offner ${ }^{1}$, Marco Abeßer $^{1}$, Melanie Ullrich ${ }^{1}$, Tobias Fischer ${ }^{1}$, Barbara Bayer ${ }^{4}$, Helga Wagner ${ }^{4}$, Stepan Gambaryan ${ }^{5,6}$, Jeanine A Ursitti ${ }^{7}$, Ibrahim M Adham ${ }^{8}$, Wolfgang A Linke',

Stephan M Feller ${ }^{10,11}$, Ingrid Fleming ${ }^{2}$, Thomas Renné ${ }^{2,13}$, Stefan Frantz ${ }^{4}$, Andreas Unger ${ }^{9}$ and Kai Schuh ${ }^{*}$

\begin{abstract}
Background: In the heart, cytoplasmic actin networks are thought to have important roles in mechanical support, myofibrillogenesis, and ion channel function. However, subcellular localization of cytoplasmic actin isoforms and proteins involved in the modulation of the cytoplasmic actin networks are elusive. Mena and VASP are important regulators of actin dynamics. Due to the lethal phenotype of mice with combined deficiency in Mena and VASP, however, distinct cardiac roles of the proteins remain speculative. In the present study, we analyzed the physiological functions of Mena and VASP in the heart and also investigated the role of the proteins in the organization of cytoplasmic actin networks.

Results: We generated a mouse model, which simultaneously lacks Mena and VASP in the heart. Mena/NASP double-deficiency induced dilated cardiomyopathy and conduction abnormalities. In wild-type mice, Mena and VASP specifically interacted with a distinct all-Spectrin splice variant (SH3i), which is in cardiomyocytes exclusively localized at Z- and intercalated discs. At Z- and intercalated discs, Mena and $\beta$-actin localized to the edges of the sarcomeres, where the thin filaments are anchored. In Mena/NASP double-deficient mice, $\beta$-actin networks were disrupted and the integrity of Z- and intercalated discs was markedly impaired.

Conclusions: Together, our data suggest that Mena, VASP, and all-Spectrin assemble cardiac multi-protein complexes, which regulate cytoplasmic actin networks. Conversely, Mena/VASP deficiency results in disrupted $\beta$-actin assembly, Z- and intercalated disc malformation, and induces dilated cardiomyopathy and conduction abnormalities.
\end{abstract}

Keywords: Actin, Heart, Mena/VASP, Spectrin, Dilated cardiomyopathy

\section{Background}

Actin dynamics regulate a tremendous range of cellular functions. Currently, it is believed that different actin isoforms and their associated actin-binding proteins form multiple specialized actin networks, which accomplish the large diversity of tasks [1]. In the heart,

\footnotetext{
* Correspondence: benz@vrc.uni-frankfurt.de; kai.schuh@uni-wuerzburg.de ${ }^{1}$ Institute of Physiology I, University of Würzburg, D-97070 Würzburg, Germany

${ }^{2}$ Institute for Vascular Signalling, Goethe-University Frankfurt, D-60590 Frankfurt, Germany

Full list of author information is available at the end of the article
}

cytoplasmic actin networks are thought to have important roles in mechanical support, myofibrillogenesis, and ion channel function [2] and elevated levels of cytoplasmic actin have been reported in dilated cardiomyopathy (DCM) [3]. Indeed, the $\alpha$-cardiac actin isoform is the major constituent of thin filaments in the sarcomeres, but circumstantial evidence supports the existence of a different actin isoform at Z- and intercalated discs, possibly $\beta$ - or $\gamma$-cytoplasmic actin $[2,4,5]$.

Proteins of the Enabled/vasodilator-stimulated phosphoprotein (Ena/VASP) family are important mediators 
in cytoskeleton control, linking cyclic nucleotide signaling pathways to actin assembly [6]. In the mammalian heart, two Ena/VASP proteins are predominantly expressed, mammalian Enabled (Mena) and VASP [7]. Mena and VASP increase actin filament assembly by their intrinsic polymerase and anti-capping activities [8] and the latter are impaired by the cyclic nucleotidedependent phosphorylation of the proteins [9-11]. Because of their ability to modulate distinct modes of actin organization, it has been proposed that Mena and VASP may serve as higher order regulators of the actin cytoskeleton [12]. Given their essential role in regulating actin dynamics, it was surprising that the deletion of the genes encoding Mena or VASP in mice resulted in only mild neuronal or platelet dysfunction [13-16]. One explanation for the lack of a pronounced phenotype could be the mutual functional compensation of the proteins. Consistent with this hypothesis, functional abnormalities in $\mathrm{Mena}^{-/-}$or $\mathrm{VASP}^{-/-}$mice were detected in tissues that predominantly express only one of the family members [7] and human VASP has been shown to rescue the lethal phenotype in Drosophila Ena ${ }^{-/-}$mutants [17]. Furthermore, Mena/VASP double-deficient mice die perinatally and suffer from severe defects in brain morphology [18]. However, a single allele of Mena is sufficient to rescue the embryonic lethal phenotype [13].

In cardiac muscle cells, scaffold proteins of the Spectrin family coordinate macromolecular protein complexes, which organize transmembrane signaling proteins and at the same time enable muscle cells to resist the extreme forces of contraction [19-21]. $\alpha$ II-Spectrin is abundant at the lateral plasma membrane but it is also enriched at Z- and intercalated discs close to the edges of the sarcomeres [22]. At the intercalated discs, aIISpectrin is a critical component of the transitional junction, an axial interface located where the thin actin filaments of the terminal sarcomere continue into the adherens junctions [4]. The critical role of $\alpha$ II-Spectrin in maintaining cardiac integrity is demonstrated by the fact that $\alpha$ II-Spectrin-deficient embryos die in utero and exhibit abnormal cardiac shape, cardiac dilation, and thinning of the myocardium [23]. These cardiac deformities are the likely cause of the embryonic lethality and reminiscent of the cardiac phenotypes induced by the loss of proteins that regulate actin dynamics [23]. Given that the direct interaction of VASP and $\alpha$ IISpectrin initiates $\beta$-actin filament assembly and stabilizes cell-cell contacts [10], it is tempting to speculate that $\alpha$ II-Spectrin:Mena/VASP complexes may be important for the formation and stability of cytoplasmic actin networks in the heart.

In the present study, we aimed to address cardiac functions of Mena and VASP using a combination of mouse models lacking either Mena or VASP, or both proteins in the heart. Mechanistically, we focused on the role of Mena/VASP in the organization of cytoplasmic actin networks and whether this has implications for mechanical support, myofibrillogenesis, and ion channel function in the mammalian heart.

\section{Results}

\section{Stage-dependent expression of cytoskeletal proteins in the mouse heart}

To determine the cardiac functions of Mena and VASP and the associated cytoskeletal proteins, $\alpha$ II-Spectrin and actin, we analyzed the protein levels in wild-type mouse hearts by Western blotting. For this, we generated a polyclonal anti-Mena antibody using a fusion protein that comprises the LERER region of mouse Mena for immunization and the specificity of the purified antibodies was controlled by Western blotting. Consistent with the reported apparent molecular weight of mouse Mena in Western blots [7], our antibody detected a protein band at $\sim 80 \mathrm{kDa}$, which was only seen in lysates of Mena-transfected, but not in the MOCK-transfected cells (Figure 1A, upper panel, lanes 1 and 2). We also investigated lysates of $\mathrm{CHO}-\mathrm{S}$ cells with a stable integration of GST-Mena [24]. Our Mena-specific antibody (Figure 1A, upper panel, lanes 3 and 4) as well as a GST-tag-specific antibody (middle panel, lanes 3 and 4) displayed a signal at $\sim 110 \mathrm{kDa}$, which was only visible in lysates of GST-Mena expressing cells but not in controls. Consistent with the absence of LERER repeats in VASP [6], our Mena antibody did not detect the purified VASP protein in Western blots (Figure 1B).

In the mouse heart, at least two Mena isoforms exist. The ubiquitously expressed isoform, which migrates with an apparent molecular weight of $\sim 80 \mathrm{kDa}$ and a splice variant, which is predominantly expressed in neuronal tissue and migrates at about $140 \mathrm{kDa}$ (Figure 1C). VASP migrates with an apparent molecular weight of 46 $\mathrm{kDa}$ in SDS-PAGE, but PKA-mediated phosphorylation of VASP on Ser157 induces an electrophoretic mobility shift of the protein from 46 to $50 \mathrm{kDa}$ (Figures $1 \mathrm{C}$ and D). Thus, depending on PKA activity in the heart, one or two protein bands can be observed in Western blots. We investigated protein expression in hearts of 1-week-old and adult mice under basal conditions, as well as the hearts of adult mice subjected to transverse aortic constriction (TAC) for 3 weeks to induce left-ventricular hypertrophy. The expression patterns of the investigated proteins showed a comparable trend. $\alpha$ II-Spectrin, Mena, VASP, and $\beta$-cytoplasmic actin were highly expressed in 1 -week old hearts and protein levels declined until adulthood (Figures 1C and D). Consistent with the reactivation of the fetal gene expression program, protein levels were significantly increased in hypertrophic hearts (Figure 1C). Taken together, the expression of 

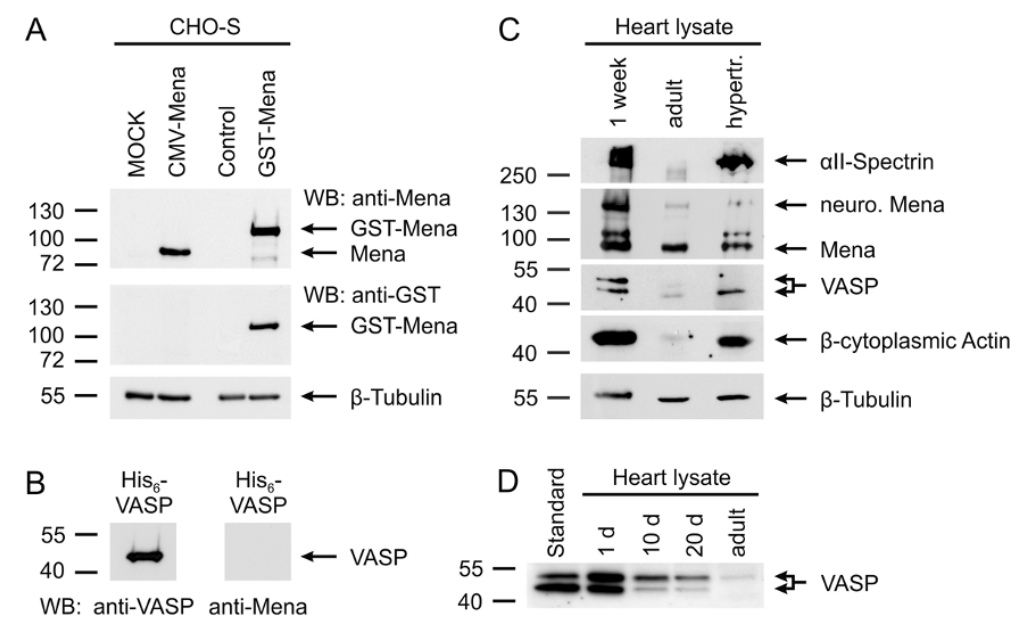

Figure 1 Stage-dependent expression of cytoskeletal proteins in the mouse heart. (A, B) Characterization of Mena-specific antibodies. (A) CHO-S cells, either transiently transfected with murine Mena (CMV-Mena) or stably transfected with GST-tagged Mena (GST-Mena), were lysed and analyzed by Western blotting with Mena-specific (upper panel) or GST-specific (middle panel) antibodies. As control, lysates of MOCK-transfected cells or lysates of CHO-S cells without stable integration of GST-Mena were run on the same gels. $\beta$-Tubulin served as loading control (lower panel). (B) 100 ng purified VASP protein was probed by Western blotting with anti-VASP (left panel) and anti-Mena (right panel) antibodies. (C, D) Stage-dependent expression of cytoskeletal proteins in the mouse heart. (C) Lysates of 1-week-old, adult, and hypertrophic adult mouse hearts were probed by Western blotting with antibodies against all-Spectrin, Mena, VASP, and $\beta$-cytoplasmic actin. Blotting for $\beta$-Tubulin was used as invariant loading control. Two Mena isoforms are expressed in the mouse heart, the general ( $80 \mathrm{kDa})$ and the neuronal-specific $(\sim 140 \mathrm{kDa})$ form. Depending on its phosphorylation state, VASP migrates at 46 or $50 \mathrm{kDa}$. (D) VASP expression in the mouse heart at postnatal day 1, 10, and 20 and in the adult heart.

Mena and VASP paralleled the expression of $\beta$ cytoplasmic actin and $\alpha$ II-Spectrin in the 1-week old, adult, and hypertrophied mouse heart.

\section{Targeted disruption of mouse Mena}

The mouse Mena gene is composed of 17 exons located on chromosome 1 (Figure 2A) and a neuronalspecific and a ubiquitous Mena protein isoform are generated by differential splicing. Both isoforms share the N-terminal Ena/VASP homology 1 (EVH1) domain, the LERER repeats, the proline-rich region (PRR), and the C-terminal EVH2 domain (Figure 2B and [25]). To target Mena gene expression, we used the murine embryonic stem (ES) cell line RRG138, in which one Mena allele has been disrupted by insertion of a gene trap vector in intron 2 (Figure 2A, black arrow and $2 \mathrm{C}$ ). Upon transcription/translation of the "trapped" Mena gene, the endogenous full-length protein is replaced by a fusion-protein encoded by the first two Mena exons and the reporter gene. Because Mena exon 3 encodes critical parts of the N-terminal EVH1 domain, a key feature of all known Mena protein isoforms, the resulting Mena/ $\beta$-geo fusion protein most likely lacks all endogenous Mena functions (Figure 2C). Following blastocyst injection of the ES cells, mice that were homozygous for the gene-trap insertion (Mena ${ }^{\mathrm{GT} / \mathrm{GT}}$ ) were generated. Mena ${ }^{\mathrm{GT} / \mathrm{GT}}$ mice were viable, fertile, and macroscopically indistinguishable from wild-type controls.

\section{Generation of Mena/VASP double-deficient mice} (Mena $^{\mathrm{GT} / \mathrm{GT}} \mathrm{VASP}^{-/-}$, dKO)

When we analyzed Mena protein levels in our Mena ${ }^{\text {GT/GT }}$ mice, we found that specifically in the brain, but not in other organs, the gene trap was "leaky" $[27,28]$, resulting in a low amount of endogenous full length protein (data not shown but compare Mena protein levels in the Mena ${ }^{\mathrm{GT} / \mathrm{GT}} \mathrm{VASP}^{-/-}$organs, Figure $3 \mathrm{~A}$, $\mathrm{dKO}$ lanes). We speculated that the residual Mena expression in the brain of Mena ${ }^{\mathrm{GT} / \mathrm{GT}}$ mice may overcome the embryonic lethal phenotype of Mena/VASP double-deficient mice and crossed the Mena ${ }^{\mathrm{GT} / \mathrm{GT}}$ animals with our $\mathrm{VASP}^{-/-}$mice [16]. Indeed, the remaining Mena protein expression in the brain was sufficient to produce viable mice (Mena ${ }^{\mathrm{GT} / \mathrm{GT}} \mathrm{VASP}^{-/-}$). In agreement with our previous studies [7], the general Mena isoform $(80 \mathrm{kDa})$ was detected in protein extracts from wild-type heart, lung, brain, and spleen. In wildtype heart and brain the antibodies also detected the neuronal splice variant (140 kDa; Figure 3A, WT lanes). In lysates from Mena ${ }^{\mathrm{GT} / \mathrm{GT}} \mathrm{VASP}^{-1-}$ mice, Mena expression was nearly undetectable in heart, lung, and spleen (Figure 3A, dKO lanes). In contrast, in brain protein extracts from Mena ${ }^{\mathrm{GT} / \mathrm{GT}} \mathrm{VASP}^{-/-}$mice, both Mena isoforms were only moderately decreased (by approximately $70 \%$ ) versus protein levels in wild-type mice (Figure 3A). We could not detect VASP protein expression in any organ from Mena ${ }^{\mathrm{GT} / \mathrm{GT}} \mathrm{VASP}^{-/-}$mice (Figure 3B). 

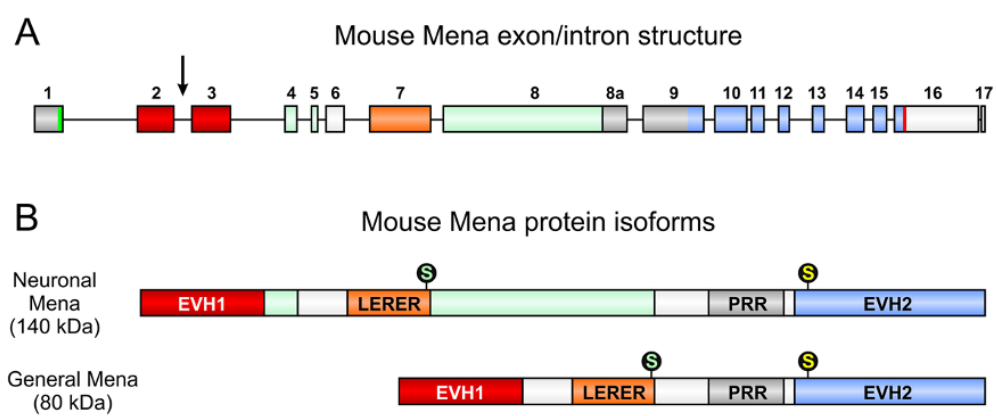

C

"Trapped" Mena allele

Fusion mRNA

Mena/ß-geo fusion protein
Targeted disruption of mouse Mena
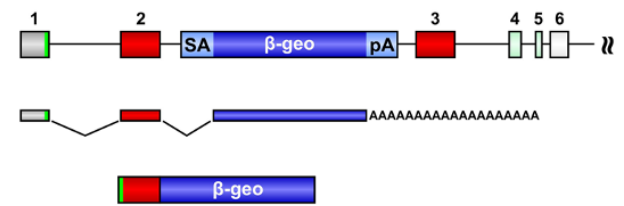

Figure 2 Targeted disruption of the mouse Mena gene. (A) The mouse Mena gene is located on chromosome 1 spanning about $115 \mathrm{~kb}$ of genomic sequence. The translational start and termination codons are given in green (exon 1) and red (exon 16), respectively. The neuronal-specific exons are shown in green. Exons 2 and 3, encoding the EnaNASP homology 1 (EVH1) domain are shown in red. Exons encoding the LERER repeats, the proline-rich region (PRR), and the EVH2 domain are indicated in orange, grey, and light blue, respectively. The insertion of a gene trap vector in intron 2 of the Mena gene is indicated by a black arrow (compare C). (B) Depending on differential splicing, a

neuronal-specific and a general Mena protein isoform exist. Mena is phosphorylated at serine 236 by PKA (green "S") and at serine 376 by PKG (yellow "S"; numbering according to the 541 aa general Mena isoform). (C) In the ES cell clone RRG138, the gene trap vector is inserted in Mena intron 2. The gene trap vector consists of a splice acceptor side (SA), a polyadenylation signal (pA), and $\beta$-geo, which encodes $\beta$-galactosidase and neomycin resistance [26]. Insertion of the gene trap vector disrupts the Mena gene and results in the expression of a Mena/ $\beta$-geo fusion protein. Because critical parts of the $\mathrm{N}$-terminal EVH1 domain are missing in the fusion protein, Mena/ $\beta$-geo most likely lacks all known endogenous Mena functions.
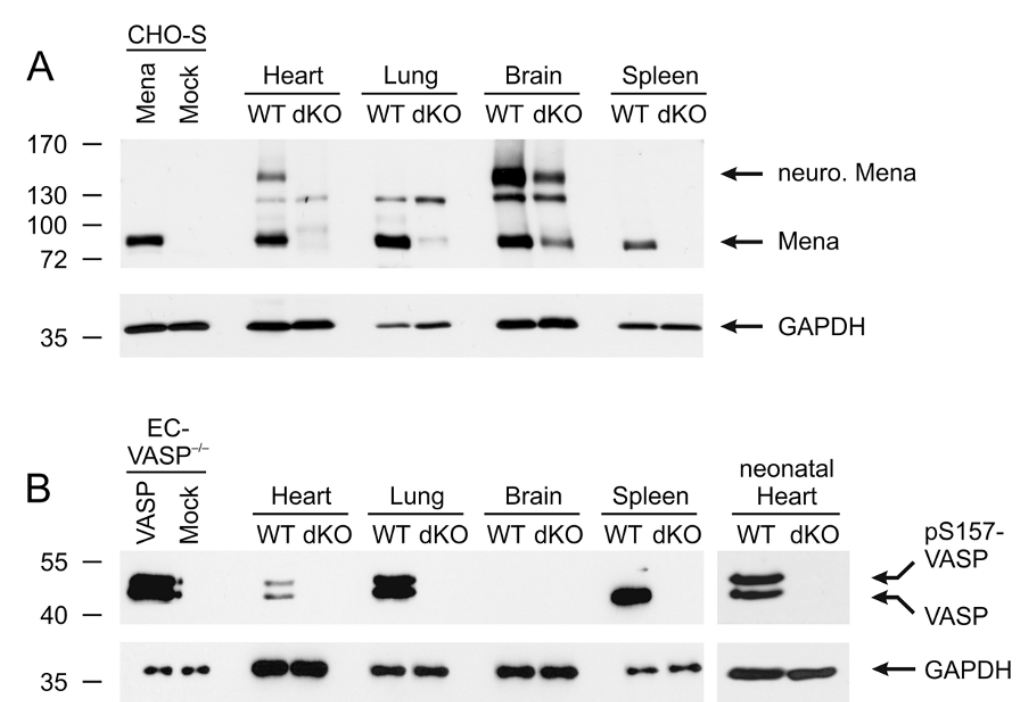

Figure 3 Mena/VASP expression in wild-type and Mena ${ }^{\mathrm{GT} / \mathrm{GT}} \mathrm{VASP}^{-/-}$mouse organs. Western blots to analyze Mena (A) and VASP (B) protein levels in heart, lung, brain, and spleen of wild-type (WT) and Mena ${ }^{G T / G T} V_{A S P}{ }^{-/-}$(dKO) mice. GAPDH and lysates of Mena- or VASP-transfected cells were used as controls. (A) The general Mena protein isoform ( $\sim 80 \mathrm{kDa})$ is present in all analyzed organs, the neuronal splice variant $(\sim 140 \mathrm{kDa})$ is only detected in heart and brain. Whereas Mena protein in dKO brain is moderately reduced to $30 \%$ of wild-type levels, Mena is almost undetectable in heart, lung, and spleen. The $130 \mathrm{kDa}$ band seen in heart, lung, and brain likely represents a cross-reaction with an unrelated protein. (B) VASP (46 or $50 \mathrm{kDa}$, depending on phosphorylation state) expression is high in the neonatal heart and the adult lung and spleen, low in the adult heart, and nearly undetectable in the brain. No VASP protein is detected in dKO organs. 


\section{Mena/VASP double-deficiency induces cardiac fibrosis and hypertrophy}

We first investigated heart weight to tibia length (HW/ $\mathrm{TL})$ ratios in adult VASP-deficient (V-KO), Mena ${ }^{\mathrm{GT} / \mathrm{GT}}$ (M-KO), and Mena ${ }^{\mathrm{GT} / \mathrm{GT}} \mathrm{VASP}^{-/-}(\mathrm{dKO})$ animals. The $\mathrm{HW} / \mathrm{TL}$ ratio of VASP-deficient mice was undistinguishable from wild-type controls and although the ratio of the Mena ${ }^{G T / G T}$ mice was slightly elevated, this trend did also not reach significance. In contrast to the individual knockouts, the HW/TL ratio of the Mena ${ }^{\mathrm{GT} / \mathrm{GT}_{\mathrm{VASP}}} \mathrm{VA-}^{-/}$ animals was significantly increased by over $20 \%$ compared to wild-type controls (Figure 4A and B).

Histological analyses of Mena ${ }^{\mathrm{GT} / \mathrm{GT}} \mathrm{VASP}^{-/-}$hearts revealed substantial dilation of both ventricles compared to wild-type hearts (Figure 4C and D), indicating eccentric hypertrophy. Dilated ventricles were not observed in the Mena ${ }^{\mathrm{GT} / \mathrm{GT}}$ or $\mathrm{VASP}^{-/-}$mice (compare end-diastolic areas in echocardiography, Figure $5 \mathrm{C}$ ). In the Mena ${ }^{\mathrm{GT} / \mathrm{GT}} \mathrm{VASP}^{-/-}$ mice, cardiac dilation was also associated with marked interstitial fibrosis (Figure 4E and F).

Consistent with cardiac fibrosis and hypertrophy, cross sectional areas of cardiomyocytes were significantly elevated in the Mena ${ }^{\mathrm{GT} / \mathrm{GT}} \mathrm{VASP}^{-/-}$animals (Figure 4G) as were mRNA levels of the cardiac hypertrophy markers; $\beta$-myosin heavy chain and atrial natriuretic peptide, and the fibrosis markers; $\alpha$-smooth muscle actin, collagen I and collagen III (Figure 4H).

\section{Mena/VASP double-deficiency induces DCM and conduction abnormalities}

Decompensated eccentric hypertrophy and cardiac dilation are characterized by a reduced functional performance of the heart [29]. Therefore, we investigated left ventricular function by echocardiography under basal conditions. M-mode and two-dimensional recordings at the mid-papillary muscle level revealed clear differences between wild-type and mutant mice. The left ventricles of Mena ${ }^{\mathrm{GT} / \mathrm{GT}}, \mathrm{VASP}^{-/-}$and Mena ${ }^{\mathrm{GT} / \mathrm{GT}} \mathrm{VASP}^{-/-}$animals displayed significantly increased end-systolic areas compared to wild-type controls (Figure 5A and B). However, the Mena ${ }^{\mathrm{GT} / \mathrm{GT}}$ and Mena ${ }^{\mathrm{GT} / \mathrm{GT}} \mathrm{VASP}^{-/-}$animals were more affected than the $\mathrm{VASP}^{-/-}$mice. A significant increase in the end-diastolic area was only observed in the Mena ${ }^{\mathrm{GT} / \mathrm{GT}} \mathrm{VASP}^{-/-}$mice (Figure $5 \mathrm{~A}$ and $\mathrm{C}$ ) and is in agreement with the histological data (see Figure 4). Consistent with an impaired cardiac contraction, Mena ${ }^{\mathrm{GT} / \mathrm{GT}} \mathrm{VASP}^{-/-}$mice displayed a significantly reduced fractional shortening compared to wild-type animals (WT 61.4\% vs. dKO 53.0\%, ${ }^{*} \mathrm{P}<0.05, \mathrm{n}=13$ ). To directly assess hemodynamic parameters in wild-type and mutant animals, we analyzed left ventricular pressures by invasive catheterization. Consistent with the occurrence of dilated ventricles, the end-systolic pressure was significantly reduced in the Mena ${ }^{\mathrm{GT} / \mathrm{GT}} \mathrm{VASP}^{-/-}$but not in the wild-type, Mena ${ }^{\mathrm{GT} / \mathrm{GT}}$, or $\mathrm{VASP}^{-/-}$animals (Figure 5D). Rates of left ventricular pressure increase were not significantly different between the groups (data not shown). Consistent with the pronounced interstitial fibrosis, however, the rate of left ventricular pressure decline was diminished in Mena ${ }^{\mathrm{GT} / \mathrm{GT}} \mathrm{VASP}^{-/-}$mice (Figure 5E).

Next, we investigated the impact of Mena/VASP deficiency on the electrical conduction in the mammalian heart and performed high resolution ECG recordings (Figure 6). Although heart rates were nearly identical in all strains (wild-type $441 \mathrm{bpm}, \mathrm{VASP}^{-1-} 434$, Mena ${ }^{\mathrm{GT} / \mathrm{GT}}$ $\left.432 \mathrm{bpm}, \mathrm{Mena}^{\mathrm{GT} / \mathrm{GT}} \mathrm{VASP}^{-1-} 439 \mathrm{bpm}\right), \mathrm{PQ}$ and QRS intervals, which reflect the intra-atrial and the intraventricular propagation of electrical signals, respectively, were significantly prolonged in the Mena ${ }^{\mathrm{GT} / \mathrm{GT}}, \mathrm{VASP}^{-/-}$, and Mena ${ }^{\mathrm{GT} / \mathrm{GT}} \mathrm{VASP}^{-/-}$mice. Interestingly, the differences in the Mena ${ }^{\mathrm{GT} / \mathrm{GT}} \mathrm{VASP}^{-/-}$animals were statistically indistinguishable from those observed in the Mena ${ }^{\mathrm{GT} / \mathrm{GT}}$ and $\mathrm{VASP}^{-/-}$mice (Figure 6).

\section{Mena is localized at Z- and intercalated discs of mouse cardiomyocytes}

Circumstantial evidence suggests that cytoplasmic actin networks are present at Z- and intercalated discs of cardiomyocytes with implications for mechanical support, myofibrillogenesis and ion channel function $[1,2,4,5]$. To determine whether or not the regulation of cytoplasmic actin dynamics at Z- and intercalated discs may be exerted through Mena and VASP, we investigated the sub-cellular distribution of the proteins by confocal microscopy and initially focused on Mena, which is more robustly expressed in the adult heart. In adult wild-type hearts, a fraction of Mena was localized at Z-discs, where it colocalized with CapZ, a protein that acts as a barbed end capping protein for $\mathrm{F}$ actin and that is found at the end of the thin filaments in the Z-disc (Figure 7A). The majority of Mena, however, was detected at intercalated discs, where it at least partially colocalized with Connexin 43 , a critical component of the gap junctions, which carry the electrical stimulus from cell to cell (Cx43, Figure 7B). To exclude channel crosstalk and sectioning artifacts, we also analyzed the subcellular distribution of Mena in isolated murine cardiac myocytes. Consistent with the findings from the intact heart, Mena was localized to Z-discs of isolated cardiomyocytes as well as to cell termini, which correspond to the intercalated discs in heart tissue (Figure 7C).

Conventional confocal microscopy has a limited axial resolution of $>200 \mathrm{~nm}$. Thus it is difficult to distinguish whether the proteins detected are truly Z-disc proteins or simply associated with nearby structures, i.e. Ttubules. To more accurately determine the subcellular distribution of Mena, we subjected ultrathin sections of 

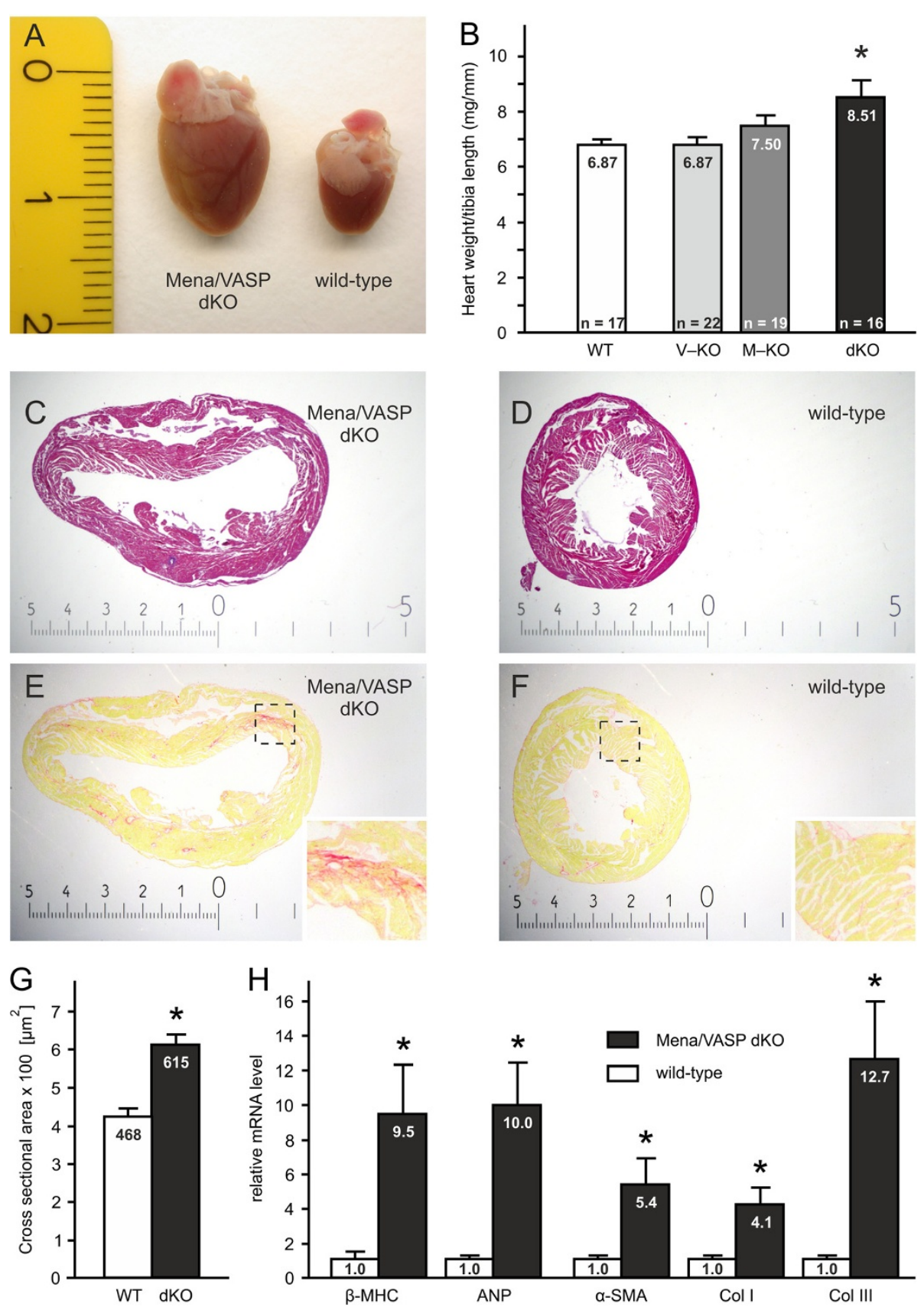

Figure 4 Enlarged and dilated hearts in Mena ${ }^{\mathrm{GT} / \mathrm{GT}} \mathrm{VASP}^{-/-}$mice. Mena ${ }^{\mathrm{GT} / \mathrm{GT}} V \mathrm{VASP}^{-/-}$(dKO) animals display macroscopically enlarged hearts $(\mathbf{A}$, photography, scale in $\mathrm{cm}$ ) and significantly increased heart weight to tibia length ratios as compared to wild-type (WT) controls (B; ANOVA, $\left.{ }^{*} \mathrm{P}<0.05\right)$. (C, D) Hematoxilin and eosin stained cross sections of Mena ${ }^{\mathrm{GT} / G T} \mathrm{VASP}^{-1-}$ (C) and wild-type (D) hearts, demonstrating dilated ventricles in the double-deficient animals. (E, F) Picrosirius red stained heart cross sections of $\mathbf{d K O}(\mathbf{E})$ and $\mathbf{W T}(\mathbf{F})$ mice to visualize collagen fibers/interstitial fibrosis. Magnified views of the indicated areas are shown as insets. The scales in images $\mathbf{C}-\mathbf{F}$ are given in $\mathbf{m m}$. (G) Cross sectional areas of cardiomyocytes from WT and Mena ${ }^{\mathrm{GT} / \mathrm{GT}} \mathrm{VASP}^{-/-}$hearts quantified by morphometric analyses (five animals per group, 25 cardiomyocytes per animal, *P<0.05). (H) Quantitative real-time RT-PCR revealed increased mRNA levels of cardiac hypertrophy markers ( $\beta$-myosin heavy chain, $\beta-M H C$; atrial natriuretic peptide, ANP) and fibrosis markers (a-smooth muscle actin, a-SMA; collagen I, Col I; and collagen III, Col III; ${ }^{*}<<0.05 ; n=6$ ) in dKO mice vs. WT controls.

murine hearts to electron microscopy (Figure $8 \mathrm{~A}$ and $\mathrm{B}$ ). Consistent with the results of the confocal microscopy studies, a fraction of Mena immunoreactivity was detected at Z-discs and the gold particles especially localized towards the edges of the adjacent sarcomeres (Figure 8A). The strongest Mena immunogold signal, however, was 

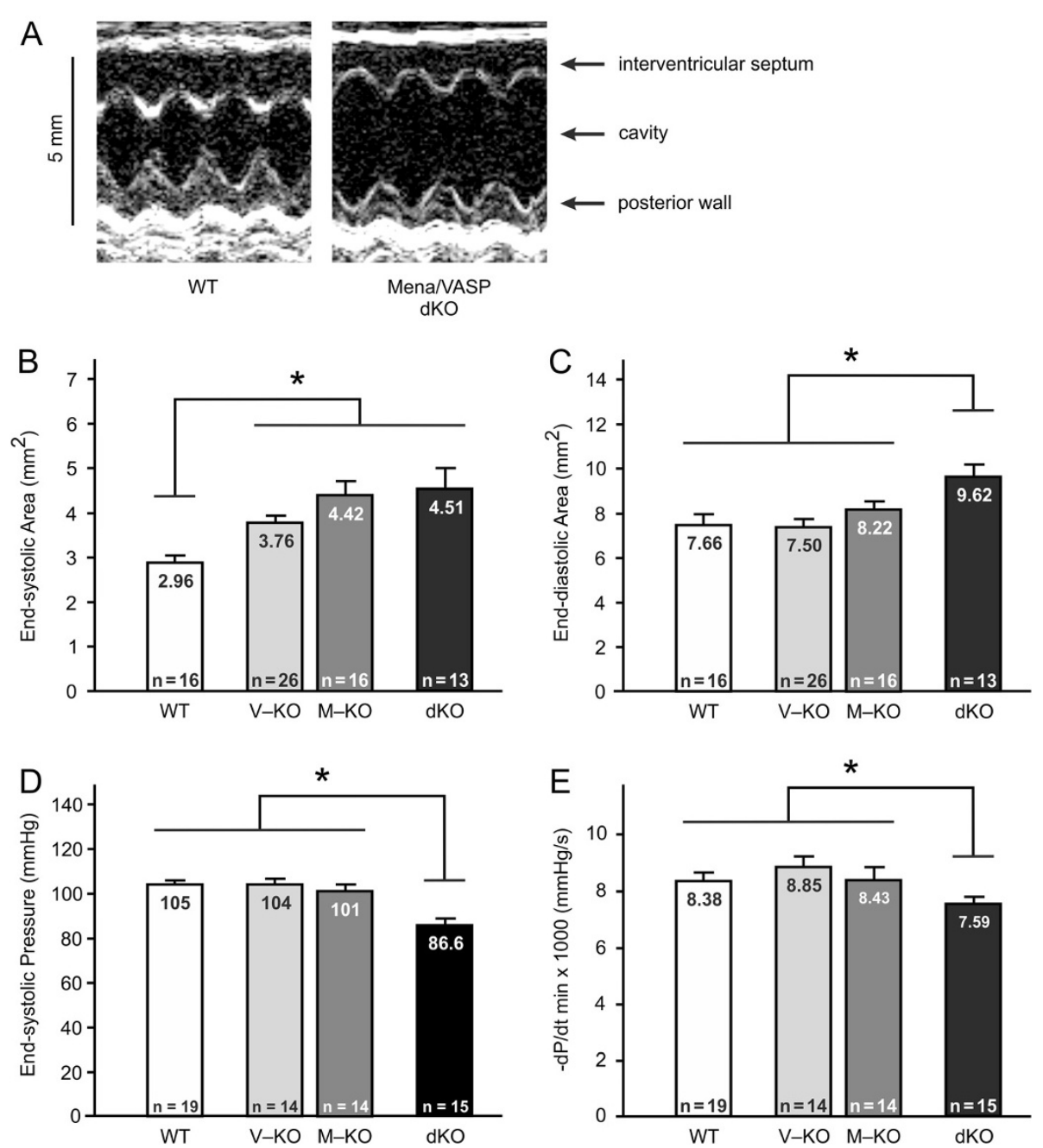

Figure 5 Mena/VASP double-deficiency induces dilated cardiomyopathy. (A-C) Echocardiography. (A) Representative M-mode recordings at the mid-papillary muscle level of wild-type (WT) and Mena ${ }^{G T / G T} V A S P^{-1-}(d K O)$ mice. (B, C) Statistical analyses of the echocardiographic measurements demonstrated a significantly increased left intraventricular end-systolic area in $\mathrm{VASP}^{-1-}(\mathrm{V}-\mathrm{KO}), \mathrm{Mena}^{\mathrm{GT} / G T}$

$(\mathrm{M}-\mathrm{KO})$, and Mena ${ }^{\mathrm{GT} / \mathrm{GT}} \mathrm{VASP}^{-/-}(\mathrm{dKO})$ animals as compared to wild-type controls (B). The left intraventricular end-diastolic area was only significantly increased in the dKO animals (C). (D, E) Left ventricular catheterization revealed a significantly reduced end-systolic pressure (D) and a significantly reduced rate of pressure decline $\mathbf{( E )}$ in the left ventricle of dKO mice. Bars represent the mean \pm S.E.M (ANOVA, $\left.{ }^{*} \mathrm{P}<0.05\right)$.

observed at the intercalated disc while the plasma membrane itself was not labeled. Indeed the majority of gold particles accumulated within the cytoplasmic edge of the dark plaque material that coats the membranes where the thin filaments of the terminal sarcomeres lead into the adherens junctions (Figure 8B). We also investigated the subcellular distribution of cytoplasmic actin isoforms. Similar to Mena, $\beta$-cytoplasmic actin was also detected at Z- and intercalated discs. At $Z$-discs, however, $\beta$-actin labeling was not limited to the edges of sarcomeres, but instead was almost evenly distributed, indicating that $\beta$-actin is an integral component of the Z-discs. At intercalated discs, again most gold particles localized to the edges of the adjacent sarcomeres and closely matched the sub-cellular distribution of Mena (Figure $8 \mathrm{C}$ and $\mathrm{D})$. In contrast to Mena and $\beta$-actin, $\gamma$-actin was neither detectable at the Z-discs nor within the I-bands of the sarcomeres. Instead, most gold particles were observed at the mitochondria in the vicinity of I-Z-I bands in cardiac myofibrils. High power magnifications suggested that $\gamma$-actin is an integral part of the filamentous structures, which project from the upper and lower terminals of Z-discs towards the mitochondria (Figure 8E). Weak immunoreactivity with small gold particle clusters was also detected inside the intercalated discs, likely representing $\gamma$-actin association with local microcompartments. In contrast to Mena and $\beta$-actin, however, no $\gamma$-actin labeling was detected at the edges of the terminal sarcomeres (Figure 8F). 

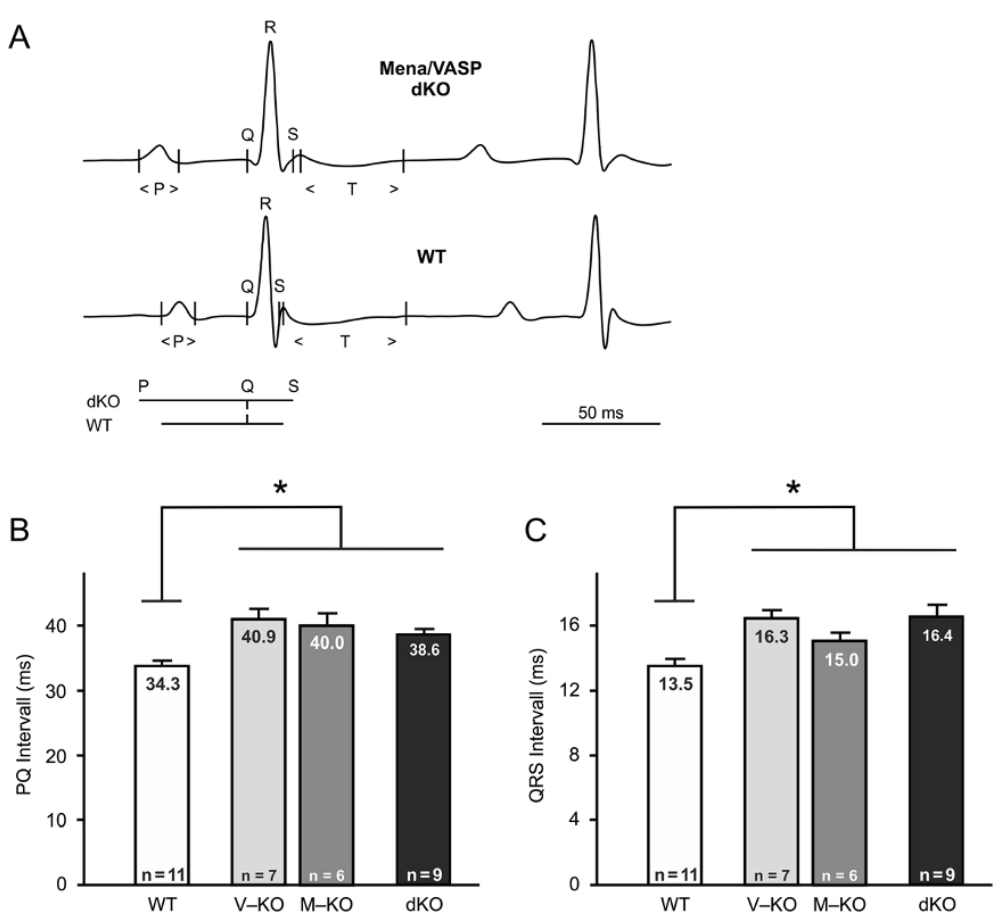

Figure 6 Mena/VASP double-deficiency results in conduction abnormalities. Electrocardiography (ECG). (A) Original, high resolution lead I ECG recording of a representative Mena ${ }^{\mathrm{G} / G T} V_{A S P}{ }^{-1-}$ (dKO, upper panel) and wild-type (WT, lower panel) mouse. Q, R, and S spikes and the P- and T-waves are indicated. A comparison of the PQ and QRS intervals from dKO and WT mice is shown below the ECG recording of the WT. (B, C) Statistical analysis of the ECG recordings demonstrated significantly prolonged PQ (B) and QRS (C) intervals in VASP ${ }^{-/-}\left(V^{-K O}\right), M^{-}$Mena ${ }^{G T / G T}$ $(\mathrm{M}-\mathrm{KO})$, and Mena ${ }^{\mathrm{GT} / G T} \mathrm{VASP}^{-/-}(\mathrm{dKO})$ mice. Bars represent the mean \pm S.E.M, one-way analysis of variance $\left.(\mathrm{ANOVA}),{ }^{*} \mathrm{P}<0.05\right)$.

Mena and VASP specifically interact with $\mathrm{SH} 3 i$, an allSpectrin splice variant

$\alpha$ II-Spectrin-deficient mice die from severe cardiac abnormalities that are reminiscent of phenotypes observed following the loss of proteins that regulate actin dynamics, such as Mena and VASP [23]. Thus, the cardiac phenotype observed in $\alpha$ II-Spectrin-deficient mice may, at least in part, be related to the failure to complex with Mena and VASP at Z- and intercalated discs. To test this hypothesis, we compared the sub-cellular distribution of Mena with that of $\alpha$ II-Spectrin and the $\alpha$ II-Spectrin splice variant; $\mathrm{SH} 3 i$, which contains a 20-amino acid insertion C-terminal to the $\mathrm{SH} 3$ domain and is also present in the mouse heart [30]. $\alpha$ II-Spectrin was found enriched at the lateral plasma membrane as well as at Z- and intercalated discs, where it colocalized with Mena and F-actin (Figure 9A). More detailed analyses of protein distribution revealed a doublet appearance of Mena and $\alpha \mathrm{II}-$ Spectrin across the intercalated disc (Figure 9A) and is consistent with co-localization of the proteins at the transitional junction, a sharp boundary that lies between the last sarcomere and the intercalated disc thin filaments $[4,22]$. In contrast, the $\mathrm{SH} 3 i$ splice variant was exclusively found at Z- and intercalated discs and not at the lateral plasma membrane. Therefore, SH3i closely paralleled the subcellular distribution of Mena and indeed the proteins displayed a high degree of colocalization at Z- and intercalated discs (Figure 9B), indicating that $\mathrm{SH} 3 i$ may be important for Mena targeting or function in the heart. Consistently, quantitative immunocolocalization analysis [31] revealed significantly higher Pearson's correlation and Mander's overlap coefficients of Mena versus SH3i than Mena versus total $\alpha$ II-Spectrin (Pearson's 0.73 versus 0.65 ; Mander's 0.94 versus $0.85, \mathrm{n}=24$, ${ }^{*} \mathrm{P}<0.05$ ). Importantly, colocalization of Mena and SH3i with F-actin was limited to Z- and intercalated discs and no colocalization with sarcomeric F-actin was observed, indicating that the association of Mena and $\mathrm{SH} 3 i$ with the $\alpha$-cardiac actin fibers of the contractile machinery is minor (Figure 9B).

Next, we compared binding of Mena/VASP to general $\alpha \mathrm{II}-$ Spectrin and the SH3i isoform. The difference between $\alpha \mathrm{II}-$ Spectrin and the SH3i isoform is a 20 amino acid insertion C-terminal to the SH3 domain in the SH3i (Figure 10A). To determine whether or not this insertion could alter $\alpha \mathrm{II}$-Spectrin binding to Mena and VASP, we generated, expressed, and purified a series of GST-fusion proteins (Figure $10 \mathrm{~B}$ and $\mathrm{C}$ ). In addition to GST alone, which served as negative control, these GST-fusion proteins included the alternatively spliced 20 amino acids (A), the $\mathrm{SH} 3$ domain (SH3), the $\alpha 9$ repeat of $\alpha \mathrm{II}-$ Spectrin $(\alpha 9)$, and the $\alpha 9$ repeat of $\mathrm{SH} 3 i$, which includes the alternatively spliced 20-amino acids ( $\alpha 9 \mathrm{~A})$. In GST pull-down 

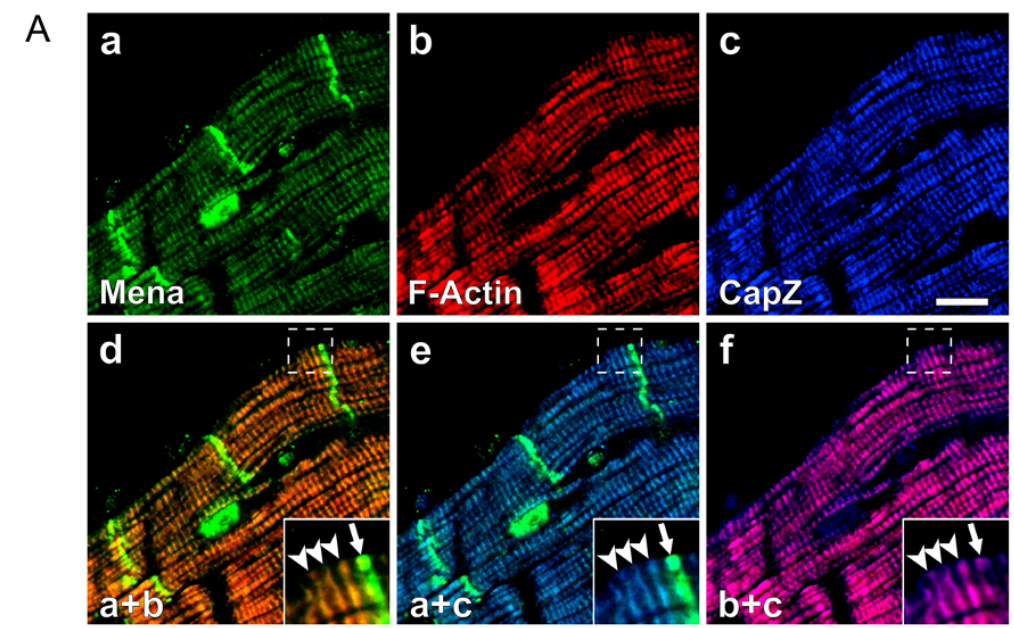

B
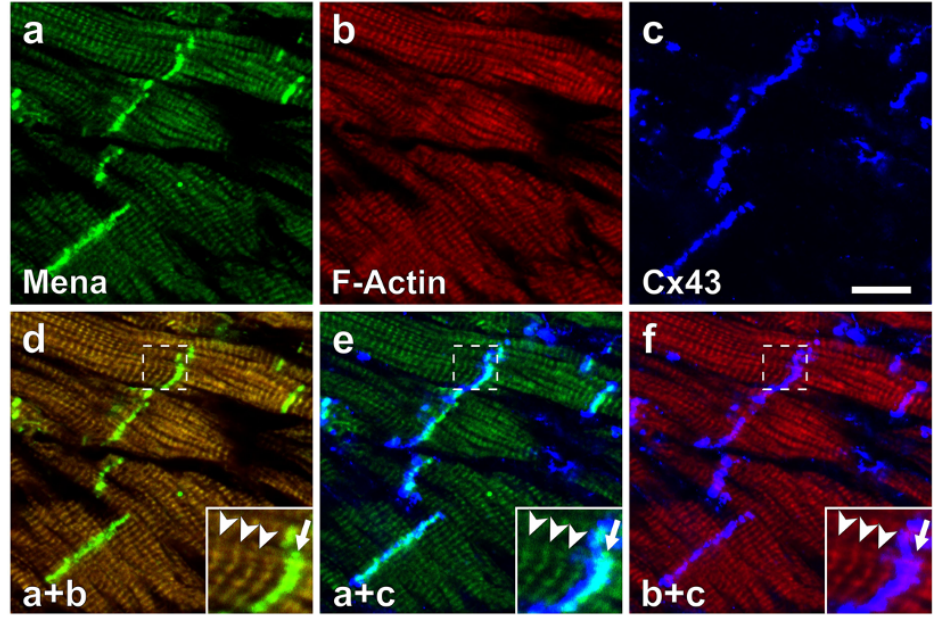

C
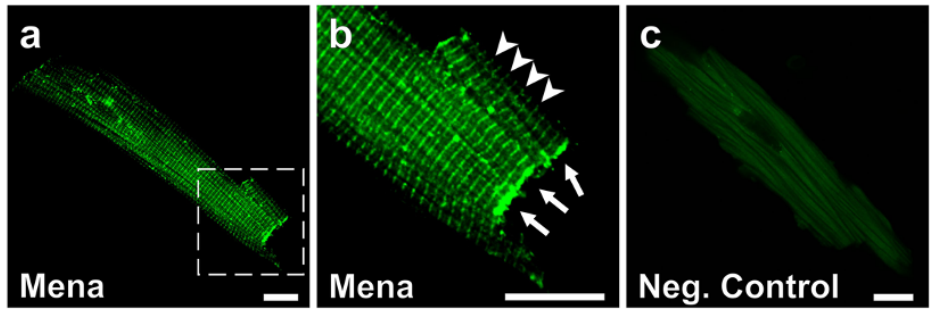

Figure 7 Mena is localized at Z- and intercalated discs of mouse cardiomyocytes. (A, B) Confocal microscopy images of adult mouse heart cryosections stained for Mena (a, green), non-isoform selective F-actin (b,red), and CapZ (A, image $\mathbf{c}$, blue) or Connexin 43 (CX43, B, image $\mathbf{c}$, blue). Mena and F-actin colocalized with CapZ at Z-dics (A, merged images $\mathbf{d}-\mathbf{f}$, arrowheads) and with Cx43 at intercalated discs (B, merged images $\mathbf{d}$-f, arrows). (C) Adult mouse cardiomyocytes were single-stained with Mena-specific antibodies followed by fluorescence-conjugated secondary antibodies (images $\mathbf{a}$ and b). Image $\mathbf{b}$ shows a magnified view of the area indicated in image $\mathbf{a}$. Mena localized to Z-discs (arrowheads) and was strongly enriched at the cell termini (arrows). Using identical laser intensities and capture settings, the fluorescent secondary antibodies alone did not reveal any cross-striated signals (image $\mathbf{c}$, negative control). Scale bars $15 \mu \mathrm{m}$.

experiments with mouse heart lysate, $\alpha 9 \mathrm{~A}$ precipitated at least 10-fold more Mena than $\alpha 9$ or SH3 (Figure 10D). Interestingly, the 20 amino acid insert alone was not sufficient for Mena binding. To analyze VASP binding in the same assay, we performed GST pull-downs with neonatal mouse heart lysates. Similar to Mena, interaction of VASP with $\alpha 9 \mathrm{~A}$ was more prominent than the interaction with $\alpha 9$ or SH3 (Figure 10E).
The interaction between Mena/VASP and SH3i, requires a functional $\mathrm{SH} 3$ domain and is regulated by phosphorylation

To characterize the formation of the Mena/VASP: $\alpha 9 \mathrm{~A}$ complex in more detail we determined whether a functional SH3 domain was essential for binding or whether $\alpha 9 \mathrm{~A}$ forms an SH3-independent site for Ena/VASP docking. The binding of $\mathrm{SH} 3$ domains to proline-rich 

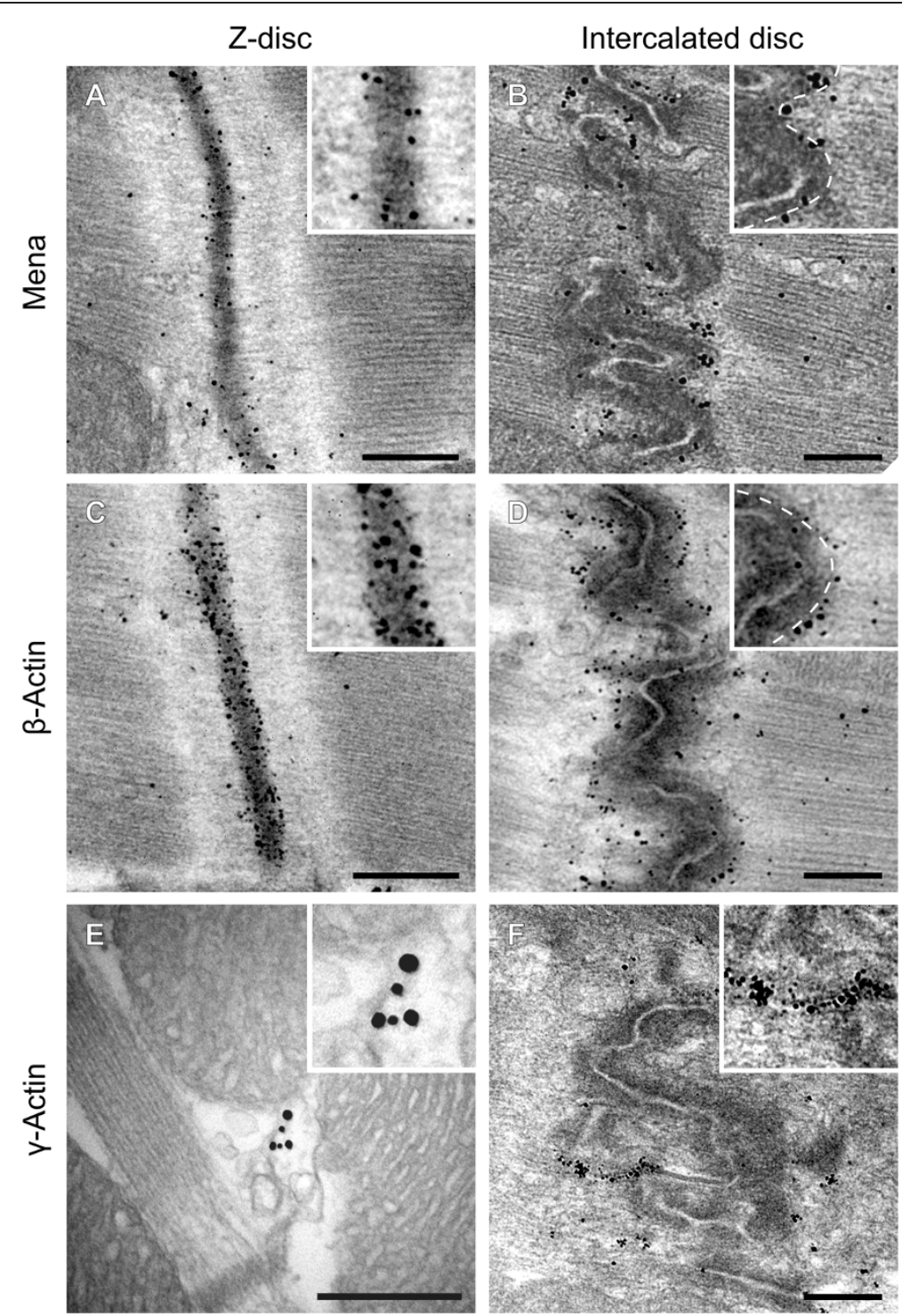

Figure 8 Mena and $\beta$-actin localize to the edges of sarcomeres at Z- and intercalated discs. Immuno-electron microscopy of Z-and intercalated disc regions in mouse papillary muscle. Sections were labelled with antibodies to Mena (A, B), $\beta$-actin (C, D), and $\gamma$-actin (E, F). The dashed line in the insets of $\mathbf{B}$ and $\mathbf{D}$ indicates the cytoplasmic edge of the dark plaque material that coats the plasma membranes where the thin filaments of the terminal sarcomeres lead into the adherens junctions. (E) Most $\gamma$-Actin immunogold labeling was observed at filamentous structures, which project from the upper and lower terminals of Z-discs towards the mitochondria. Scale bars $500 \mathrm{~nm}$.

ligands is mediated by two conserved hydrophobic pockets within the SH3 domain. To abrogate the binding of the SH3 domain, we substituted a highly conserved tryptophan residue (W1004) which is critically involved in formation of the second hydrophobic binding pocket [32], with an arginine residue to generate the W1004R point mutant. In GST pull-down experiments with mouse heart lysates, Mena binding to W1004R was markedly reduced versus binding to $\alpha 9 \mathrm{~A}$ and was comparable to the background binding to GST alone (Figure 10F). We have previously shown that the PKA-mediated phosphorylation of VASP impairs its interaction with the SH3 domain of $\alpha \mathrm{II}-$ Spectrin [10]. Because the interaction of Mena and VASP with $\alpha 9 \mathrm{~A}$ appeared to be stronger than the interaction with either $\alpha 9$ or SH3 (Figure 10D and E), we investigated whether the binding of $\alpha 9 \mathrm{~A}$ to VASP is also regulated by PKA. Therefore, purified recombinant VASP was phosphorylated with activated PKA in vitro, which resulted in the complete phosphorylation of the protein on Ser157 (Figure 10G). In subsequent pull-down assays, $\alpha 9 \mathrm{~A}$ readily precipitated the non-phosphorylated, but not the PKAphosphorylated protein (Figure 10G). 

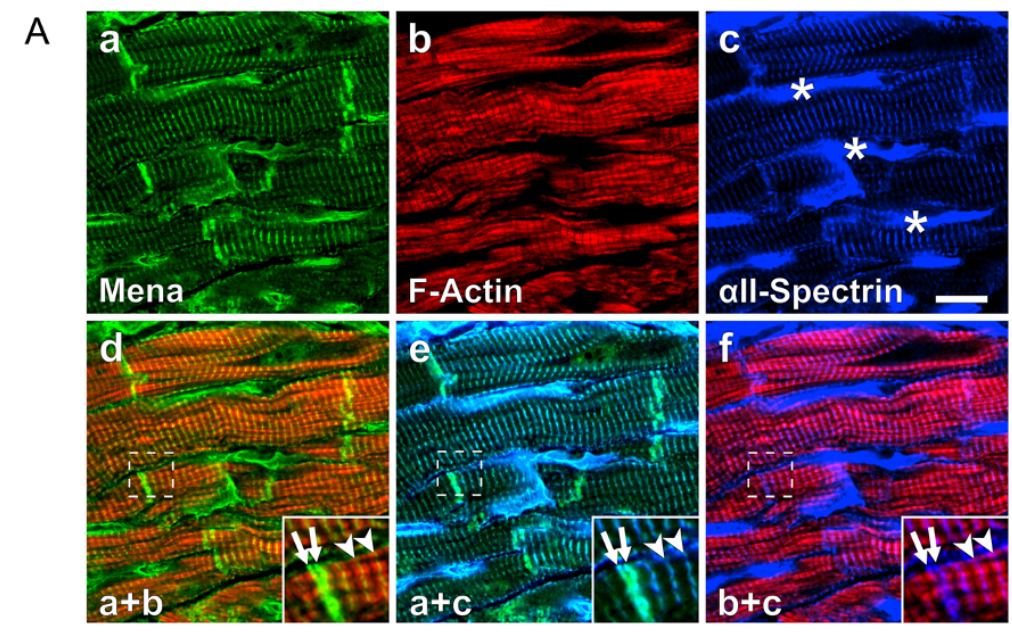

B
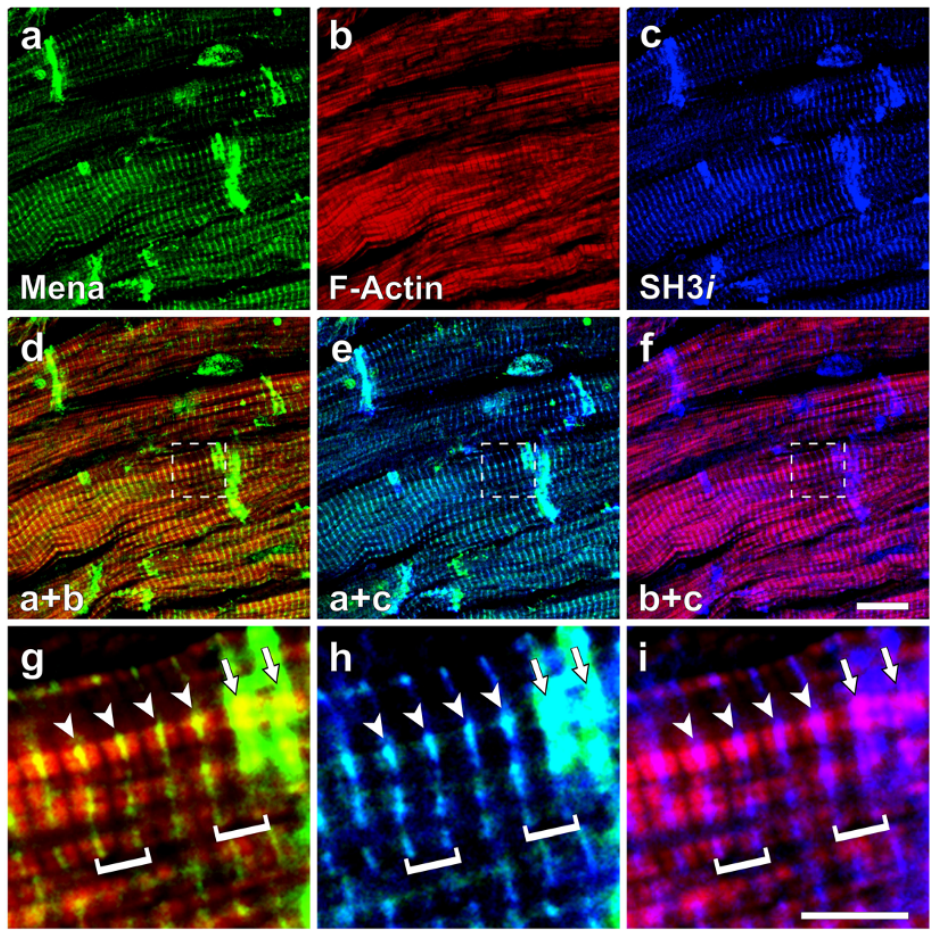

Figure 9 Mena specifically colocalizes with the all-Spectrin splice variant SH3i. (A, B) Confocal microscopy images of adult mouse heart cryosections stained for Mena (a, green), F-actin (b, red), and total all-Spectrin (A, image $\mathbf{c}$, blue) or the all-Spectrin splice variant SH3i (B, image C, blue). (A) Mena, F-actin, and a portion of total all-Spectrin colocalized at Z-discs (A, merged images $\mathbf{d}-\mathbf{f}$, arrowheads) and intercalated discs (A, merged images $\mathbf{d}$-f, arrows. Detailed analyses revealed a doublet appearance of the proteins across the intercalated disc (A, images $\mathbf{d}-\mathbf{f}$, insets). Most allSpectrin was detected at the lateral plasma membrane (A, image $\mathbf{C}$, asterisks) and colocalization with Mena and F-actin at these sites was minor. (B) SH3i was exclusively detected at Z- and intercalated discs but was absent from the lateral plasma membrane compare image $\mathbf{c}$ of $\mathbf{A}$ and $\mathbf{B}$ ). Merged images $\mathbf{d}$-f and magnified views of the indicated areas therein (images $\mathbf{g}$-i) revealed high degree of colocalization of Mena and SH3i at Z-discs (images $\mathbf{g - i}$, arrowheads) and intercalated discs (arrows), the lateral boundaries of the sarcomeres, which are exemplarily highlighted by brackets. Outside Zand intercalated discs, colocalization of Mena and SH3i with F-actin was minor. Scale bars in images c and f $15 \mu \mathrm{m}$, image i $5 \mu \mathrm{m}$.

Taken together, these data indicate that the $\alpha$ IISpectrin splice variant $\mathrm{SH} 3 i$ is the prominent Mena/ VASP interaction partner at Z- and intercalated discs. Moreover, the SH3 domain of $\mathrm{SH} 3 i$ is essential for complex formation and PKA-mediated VASP phosphorylation impairs its binding in vitro.
Mena and SH3i establish multi-protein complexes at $\mathrm{Z}$ - and intercalated discs

To determine whether $\mathrm{SH} 3 i$ and Mena form complexes in vivo and to elucidate whether or not the proteins are important structural components of Z- and intercalated discs, we performed immunoprecipitation (IP) studies from 


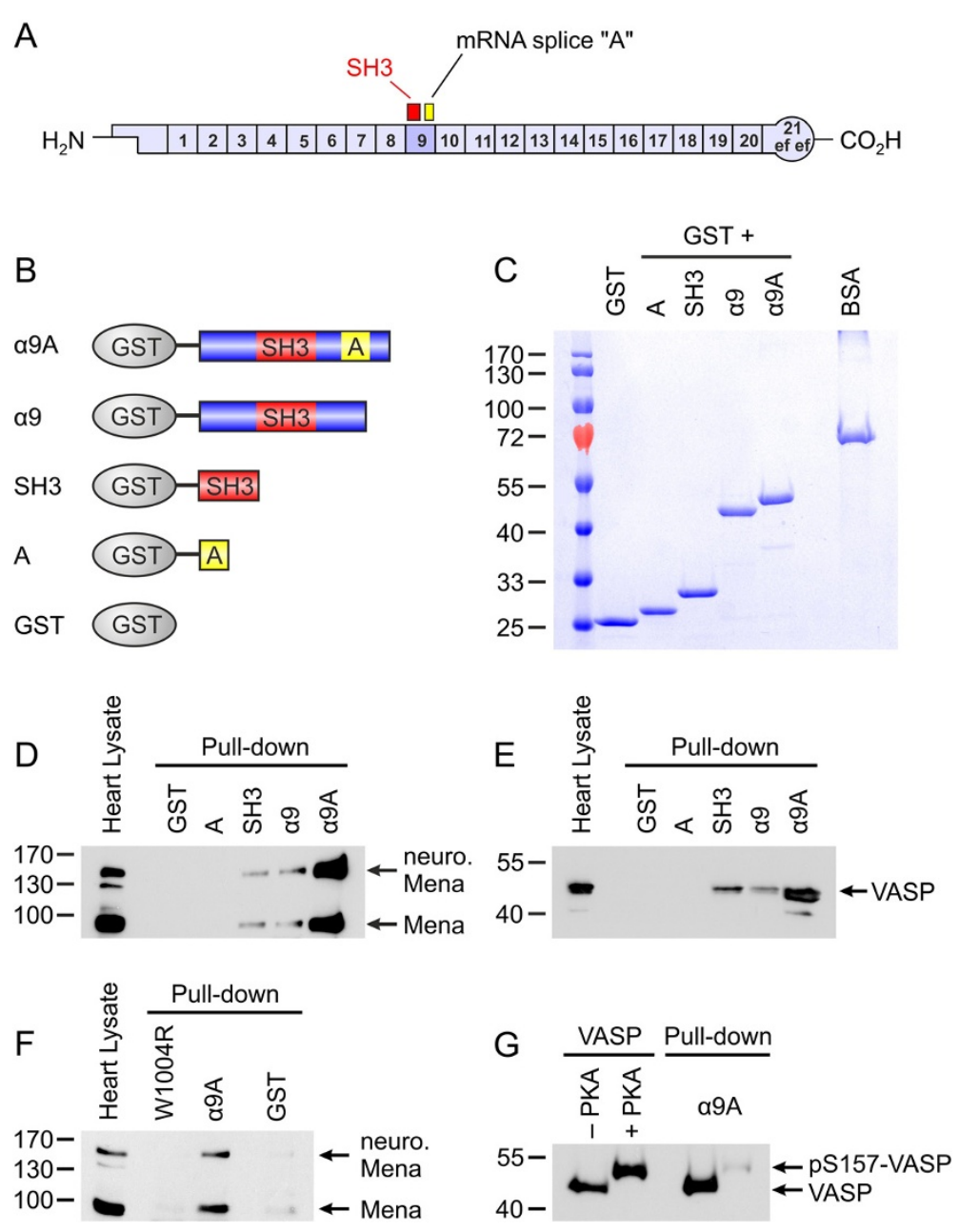

Figure 10 C-terminal extension of the all-Spectrin SH3 domain enhances interaction with Mena and VASP. (A) all-Spectrin is composed of 21 triple-helical repeats. Two all-Spectrin splice variants exist in the mouse heart, either with or without a 20-amino acids insertion in the $9^{\text {th }}$ repeat ("A"), C-terminal to the SH3 domain (indicated in red). The splice variant with the insertion is termed SH3i. (B, C) Schematic diagram (B) and Coomassie stained gel (C) of the purified GST-all-Spectrin fusion proteins used for the pull-down experiments shown in D-G. Two microgram bovine serum albumin (BSA) served to calibrate the protein load. (D, E) Lysates of adult (D) and neonatal (E) mouse hearts were incubated with the depicted GST-fusion proteins and the precipitated material was blotted with Mena- or VASP-specific antibodies, respectively. (F) The interaction between Mena and SH3i, requires a functional SH3 domain and mutation of a9A to exchange the conserved Trp1004 residue with an arginine (W1004R) abrogates the binding. (G) The interaction between purified recombinant VASP and SH3i is regulated by phosphorylation. GST-a9A readily precipitated the non-phosphorylated VASP (46 kDa), but not PKA-phosphorylated protein (pS157-VASP, 50 kDa).

lysates of adult mouse hearts. Mena-specific antibodies coprecipitated total $\alpha \mathrm{II}-\mathrm{Spectrin}$ and SH3i but the ratio of precipitate to input (heart lysate) was greater for $\mathrm{SH} 3 i$ than $\alpha \mathrm{II}-$ Spectrin (Figure 11A). Moreover, given that the antibody against $\alpha \mathrm{II}-$ Spectrin also detects the $\mathrm{SH} 3 i$ splice variant (but not vice versa) it seemed that the preferred interaction is between Mena and the SH3i splice variant. Consistently, SH3i-specific antibodies precipitated 8-fold more Mena protein than general $\alpha$ II-Spectrin-specific antibodies (Figure 11B-D). Mena was also found to complex with actin, sarcomeric $\alpha$-actinin, and $\mathrm{Cx} 43$ (Figure 11A). We also investigated the interaction of alI-Spectrin or SH3i with $\mathrm{Cx} 43$ and found that, similar to its binding to Mena,
SH3i precipitated significantly more Cx43 (6.6-fold) than $\alpha$ II-Spectrin (Figure 11 B-D). In contrast, complex formation of $\alpha$ II-Spectrin or SH3i with actin and sarcomeric $\alpha$ actinin was similar. Ena/VASP proteins associate at or near the barbed ends of actin filaments thereby restricting the access of barbed end capping proteins. In our IP studies, we investigated the association of Mena, $\alpha$ II-Spectrin, and SH3i with CapZ, an F-actin capping protein at Z-discs. Although Mena, $\alpha \mathrm{II}-$ Spectrin, and SH3i are also found at Zdiscs in cardiac myocytes, we could not detect a physical association between any of the actin binding proteins and CapZ (Figure 11), indicating a mutually exclusive binding of the proteins to actin. 

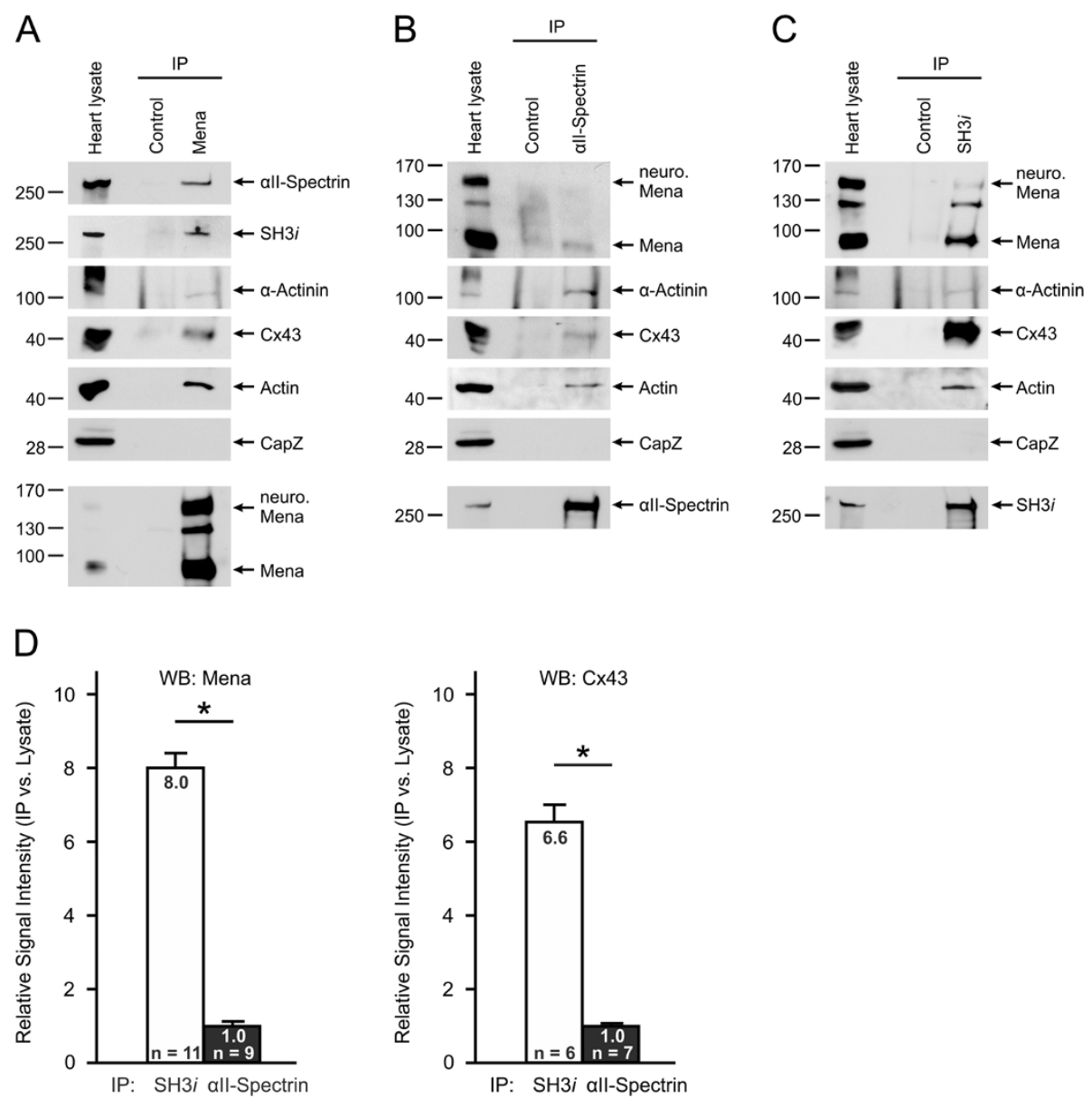

Figure 11 Mena and SH3i establish multi-protein complexes at Z- and intercalated discs. Representative Western blots of Immunoprecipiation (IP) studies. Lysates of adult mouse hearts were immunoprecipitated with Mena-specific (A), general all-Spectrin-specific (B), or SH3i-specific (C) antibodies and the precipitated material was probed with antibodies against total all-Spectrin, SH3i, Mena, actin, the Z-disC components a-Actinin and CapZ, and the intercalated disc marker Connexin 43 (CX43). Western blot analysis of the precipitated material with the same antibodies that were used for IP served as control (lowermost panel in A, B, and C). Mena, all-Spectrin, and SH3i were found in complexes with actin, a-Actinin and CX43, but not in complexes with CapZ. (D) Statistical analysis of Mena and Cx43 protein amounts in IPs with general all-Spectrin-specific (black bars) or SH3i-specific antibodies (white bars). SH3i precipitated more Mena and Cx43 than all-Spectrin. Bars represent the mean \pm S.E.M (ANOVA, $\left.{ }^{*} \mathrm{P}<0.05\right)$.

\section{Mena/VASP double-deficiency impairs Z- and intercalated disc integrity}

Our data revealed that $\alpha$ II-Spectrin/SH3i, Mena, and actin form multi-protein complexes in cardiomyocytes. To test if these complexes are important for the structural integrity of $\mathrm{Z}$ - and intercalated discs, we compared the morphology of hearts from wild-type and Mena ${ }^{\mathrm{GT} / \mathrm{GT}} \mathrm{VASP}^{-/-}$ mice by pre-embedding immunogold electron microscopy. First, we focussed on the morphology of the Z-discs and labeled thin sections with anti $\alpha$-Actinin antibodies. In sections from wild-type and Mena ${ }^{\mathrm{GT} / \mathrm{GT}} \mathrm{VASP}^{-/-}$ mice, immunogold particles were equally found at Zdiscs. However, compared to the wild-type sections, the integrity of the Z-discs in the Mena ${ }^{\mathrm{GT} / \mathrm{GT}} \mathrm{VASP}^{-/-}$ mice was markedly altered and demonstrated a wavy and fractured appearance (Figure 12A and B). The thin filaments of the contractile apparatus are anchored at Zdiscs and consistent with the ruptured Z-discs in the Mena ${ }^{\mathrm{GT} / \mathrm{GT}} \mathrm{VASP}^{-/-}$hearts, the morphology of actin filaments in the I-band also appeared disorganized and chaotic (Figure 12A and B). Next, we assessed whether Mena/VASP deficiency affects the localization of $\alpha I I-$ Spectrin/SH3i at Z-discs. In contrast to sections from wild-type hearts where the proteins were almost equally spread along the Z-disc, clusters of $\alpha \mathrm{II}-$ Spectrin and SH3 $i$ were found within the ruptured Z-discs and often associated with the emerging gaps in cardiac Z-discs from Mena ${ }^{\mathrm{GT} / \mathrm{GT}} \mathrm{VASP}^{-/-}$mice (Figure $12 \mathrm{C}-\mathrm{F}$ ). Gamma-actin labeling at the filamentous structures projecting from the Zdisc termini to the mitochondria was indistinguishable in wild-type and Mena ${ }^{\mathrm{GT} / \mathrm{GT}} \mathrm{VASP}^{-/-}$heart sections (data not shown) but the $\beta$-actin pattern was markedly altered in 


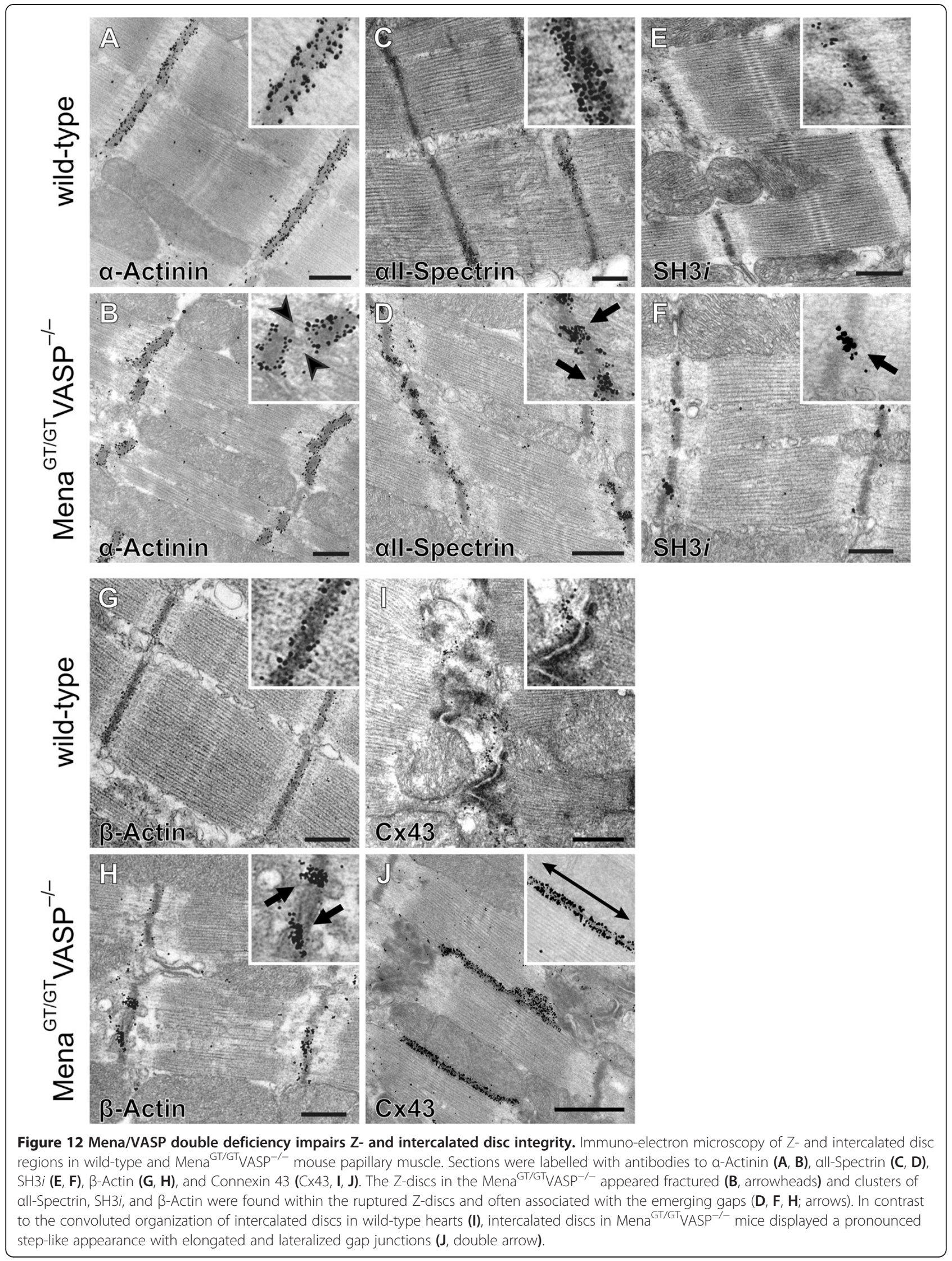


Mena ${ }^{\mathrm{GT} / \mathrm{GT}} \mathrm{VASP}^{-/-}$hearts. Similar to the $\alpha \mathrm{II}-$ Spectrin /SH3i labeling, $\beta$-actin immunogold particles clustered in the fractured Z-discs (Figure 12G and H).

Finally, we compared the morphology of intercalated discs in wild-type and Mena ${ }^{\mathrm{GT} / \mathrm{GT}} \mathrm{VASP}^{-1-}$ mice and analyzed Connexin 43 localization. Whereas cardiac muscle from wild-type mice displayed the typical convoluted organization of intercalated discs with compact gap junctions intimately connected with adjacent desmosomes and adherens junctions, intercalated discs in Mena ${ }^{\mathrm{GT} / \mathrm{GT}} \mathrm{VASP}^{-1-}$ mice displayed a pronounced steplike appearance with elongated, lateralized gap junctions. Indeed, adherens junctions at both sides of the elongated gap junctions were separated by almost the entire length of a sarcomere (Figure 12I and J).

Thus, Mena/VASP double-deficiency results in structural alteration of Z- and intercalated discs. This may explain the conduction delays and the development of the dilated cardiomyopathy in Mena ${ }^{\mathrm{GT} / \mathrm{GT}} \mathrm{VASP}^{-/-}$ mice and supports the hypothesis of an important role of $\beta$-cytoplasmic actin networks for the mechanical support, myofibrillogenesis, and ion channel function in the heart.

\section{Discussion}

In the present study, we analyzed the physiological functions of Mena and VASP in the mammalian heart and investigated the role of the proteins in the organization of cytoplasmic actin networks.

\section{Stage-dependent expression of cytoskeletal proteins in the mouse heart}

We found that cardiac VASP expression was upregulated in lysates from 1-week-old and adult hypertrophic hearts, conditions in which the heart is confronted with a sudden and dramatic increase in mechanical workload. Mena expression followed a similar trend, but the protein is more robustly expressed in the adult heart, pointing to a more specialized role of VASP in the developing or remodelling heart. The upregulation of the proteins in the infant and hypertrophied heart could partially be due to non-cardiomyocyte cells and fibrosis. However, Mena and VASP protein levels were also upregulated in cardiac myocytes isolated from mice subjected to ascending aortic constriction [33] and from the developing rat heart [7], demonstrating that the increased protein expression is likely cardiomyocytespecific. Consistent with activation of $\beta$-adrenergic signaling pathways, VASP from infant and hypertrophied hearts was frequently PKA phosphorylated and displayed its characteristic doublet on Western blots. How PKAmediated phosphorylation regulates VASP function under these conditions is currently unknown and requires further investigations.
Taken together, Mena and VASP function may be especially important in heart development and remodeling. However, additional studies on the role of the proteins in embryonic and postnatal development and in hypertrophic conditions, including transverse aortic constriction or sustained activation of $\beta$-adrenergic signaling, are required to experimentally address this hypothesis.

\section{Cardiac phenotype of Mena/VASP single- and double- deficient mice}

The present study is the first to systematically address the consequences of Mena-, VASP-, and Mena/VASP double-deficiency in cardiac physiology.

Together with the histology data, our echocardiographic and hemodynamic measurements revealed an important role of Mena and VASP for the mechanical properties of the heart. Left-ventricular performance was increasingly impaired in adult VASP-, Mena-, and Mena/VASP doubledeficient mice and only the double-deficient animals developed dilated cardiomyopathy. Consistent with a higher expression level of Mena in the adult heart, this suggests that Mena is more important for the mechanical properties of the heart than VASP. Because conventional $\mathrm{Mena}^{-/-}$mice display a cardiac phenotype, which is comparable to our Mena ${ }^{G T / G T}$ mice [34], the remaining Mena expression in the brain of our Mena ${ }^{\mathrm{GT} / \mathrm{GT}}$ mice does not seem to influence cardiac performance. With respect to cardiac mechanics, Mena and VASP can, to a certain extent, compensate for the loss of the other family member. However, the functional compensation is not sufficient to preserve full cardiac performance in the single gene-deficient animals. We investigated Mena protein levels in VASPdeficient animals and vice versa. However, consistent with our previous findings [16], we could not detect a compensatory upregulation of the proteins (data not shown).

When we investigated the electrical conduction in wild-type and mutant hearts, intra-atrial and intraventricular propagation of electrical signals was equally delayed in Mena/VASP single- and double-deficient mice. In contrast to heart mechanics, this suggests that Mena and VASP have indispensable functions in the propagation of electrical signals, likely in the formation of the gap junctions in intercalated discs.

Previously, a dominant negative strategy with cardiacspecific overexpression of the VASP-EVH1 domain was used to disrupt the in vivo function of Mena and VASP [35]. Animals with high transgene expression developed cardiac abnormalities and died within 2-6 weeks after birth [35]. Because our Mena/VASP double-deficient mice did not reveal an obviously reduced lifespan, it is likely that the transgene caused off-target effects and possibly affected other EVH1 domain-containing proteins. 


\section{Mena/VASP, SH3i, and actin assemble multi-protein complexes at Z- and intercalated discs}

Our results revealed that Mena/VASP specifically interact with a distinct $\alpha$ II-Spectrin splice variant (SH3i), which is exclusively localized at Z- and intercalated discs of cardiomyocytes. The $\mathrm{SH} 3 i$ isoform contains a 20 amino acid insertion C-terminal to the SH3 domain, which enhances the binding to Mena and VASP in pulldown assays. Similar to the isolated SH3 domain [10], PKA-mediated VASP phosphorylation abrogates binding of SH3i to VASP in vitro. However, it remains to be detailed whether activation of $\beta$-adrenergic signaling pathways affects the Mena/VASP: SH3i complex formation in vivo. Our immunoprecipitation experiments suggested that Mena, SH3i, and actin assemble multi-protein complexes at Z- and intercalated discs. Interestingly, we could not detect other actin-binding proteins such as CapZ in these complexes, arguing for the existence of distinct actin networks in cardiomyocytes. We used immunogold labeling to determine the distribution of Mena in cardiac myocytes. At Z- and intercalated discs of mouse cardiomyocytes, Mena localized to the edges of the sarcomeres, where the thin filaments are anchored. Importantly, we could show for the first time that actin filaments at these sites are, at least in part, composed of the $\beta$-cytoplasmic actin isoform. In contrast, colocalization of Mena with the thin filaments of the contractile machinery or the filamentous structures projecting from the Z-disc termini towards the mitochondria was minor. This suggests that in the adult mouse heart Mena is not involved in the regulation of $\alpha$-cardiac or $\gamma$-cytoplasmic actin fibers but specifically interacts with $\beta$-cytoplasmic actin networks. However, currently it is unknown how Mena and VASP may distinguish between different actin isoforms and two commonly held concepts may give an explanation. First, Mena and VASP could discriminate between muscle and cytoplasmic actin isoforms, which has been shown for a subset of other actin-binding proteins including Thymosin b4 and profilin [1]. All actin isoforms share at least 93\% identity and $\beta$ - and $\gamma$-actin differ by only four residues at the N-terminus. Up to now, the actin epitope, which binds to Mena or VASP, has not been identified. It is tempting to speculate that Mena and VASP may favor binding to cytoplasmic actin isoforms. Indeed most cellular effects of Mena and VASP, such as endothelial barrier function, fibroblast migration, and axon guidance, are associated with the regulation of $\beta$-actin actin dynamics $[10,11,36]$. Moreover, profilin, which directly interacts with Mena and VASP and increases the anticapping and filament elongation activities of the proteins, binds cytoplasmic actin with higher affinity than muscle actin [37]. Nevertheless, there may be a second explanation for the isoform-specific interaction of the proteins with actin. Mena and VASP may be localized to distinct subcellular regions, perhaps as a result of differential interactions with other proteins such as $\alpha \mathrm{II}-$ Spectrin. Because these interactions can be regulated by cyclic nucleotide-dependent signaling pathways, this may allow the cell to modulate actin dynamics in response to external stimuli in a spatial and temporal defined manner. In agreement with this concept, VASP readily increases the filament assembly of muscle actin in vitro $[9,11]$, arguing against an isoform-selective actin binding of VASP per se. To our knowledge, this is the first study indicating that Mena and VASP interact with actin fibers in an isoform-dependent manner.

\section{Mena/VASP double-deficiency impairs Z- and intercalated disc integrity}

When we analyzed the morphology of cardiomyocytes in our Mena ${ }^{\mathrm{GT} / \mathrm{GT}} \mathrm{VASP}^{-/-}$mice, Z-discs appeared wavy and fractured. Furthermore $\alpha \mathrm{II}-$ Spectrin, $\mathrm{SH} 3 i$, and $\beta$ actin formed clusters within the Z-discs and actin filaments of the I-band appeared disorganized. It is tempting to speculate that Mena/VASP deficiency results in a disruption of the multi-protein complexes with $\alpha$ IISpectrin and $\beta$-actin and thus impairs the structural integrity of the Z-discs, which in turn disrupts the anchoring of the thin filaments from the I-band. However, we cannot rule out that the ruptured Z-discs are the primary consequence of Mena/VASP double-deficiency and that clustering of $\alpha \mathrm{II}$-Spectrin and $\beta$-actin are secondary effects. One finding that argues against a secondary effect of $\alpha$ II-Spectrin and $\beta$-actin clustering, however, is the sub-cellular distribution of $\alpha$-Actinin in wild-type and Mena ${ }^{\mathrm{GT} / \mathrm{GT}} \mathrm{VASP}^{-/-}$mice. Although the Z-disc structure was clearly impaired in the Mena ${ }^{\mathrm{GT} / \mathrm{GT}} \mathrm{VASP}^{-/-}$mice, the $\alpha$-Actinin staining per se was indistinguishable from wildtype mice, indicating that in contrast to $\alpha$ II-Spectrin and $\beta$-actin other components of the actin network at Z-discs are not affected by the malformation of the Z-discs.

Mena/VASP double-deficiency also impaired the structural integrity of intercalated discs. In our Mena ${ }^{\mathrm{GT} / \mathrm{GT}} \mathrm{VASP}^{-/-}$ mice, especially the morphology of gap junctions was markedly changed. In the double-deficient animals, Connexin 43-positive gap junctions were excessively lateralized and elongated and clearly separated from desmosomes and adherens junctions. Currently, information how actin networks affect gap junction formation is sparse. Interactions of connexins with the actin cytoskeleton and associated proteins appear to stabilize gap junctions at the plasma membrane and $\alpha$ II-Spectrin has been shown to co-precipitate with Cx43, likely via interaction with ZO-1 [38]. Our data indicate that especially $\mathrm{SH} 3 i$ may be involved in cytoskeletal organization at gap junctions as $\mathrm{SH} 3 i$ precipitated significantly more Cx43 and Mena than the general $\alpha$ II-Spectrin 


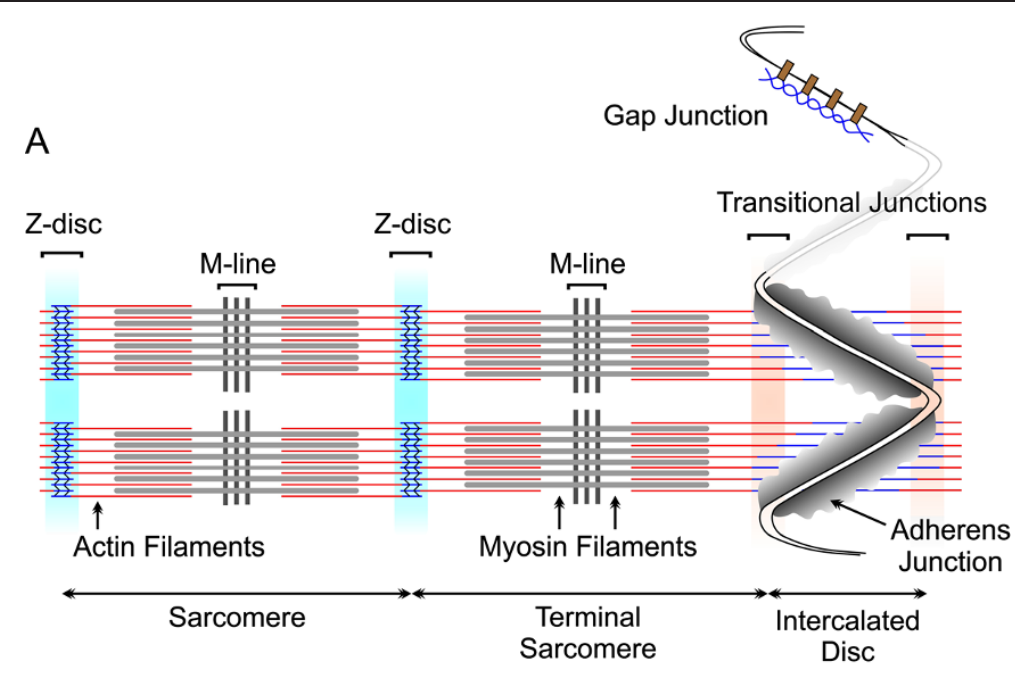

B
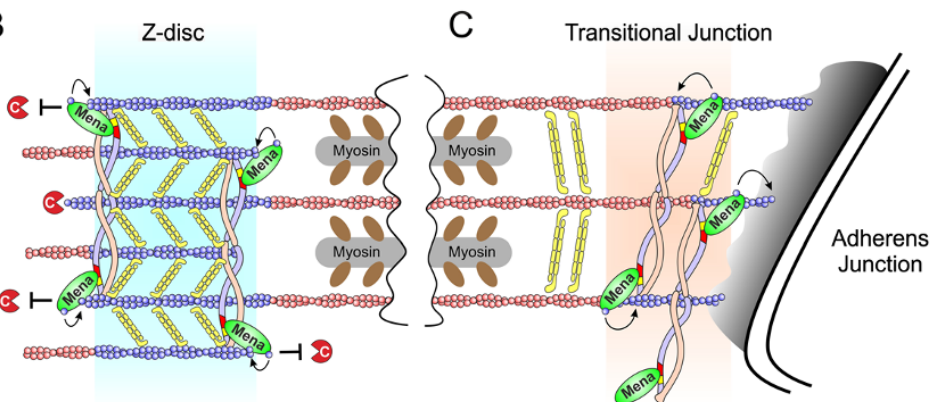

D
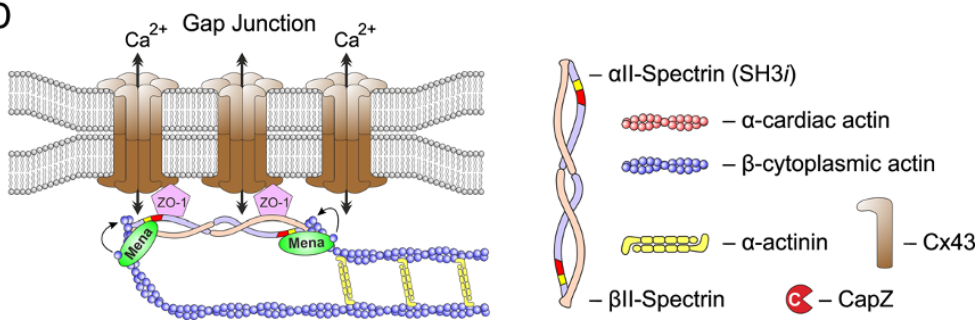

Figure 13 Proposed role of Mena/VASP and all-Spectrin for cytoplasmic actin assembly in the mammalian heart. (A) Diagram of myofibrils and junctions in two adjacent cardiomyocytes. The longitudinal force generated by sliding of actin and myosin fibers along each other is transduced between adjacent sarcomeres at the Z-disc and between adjacent cardiomyocytes at the adherens junctions of the intercalated disc. In contrast to the transverse adherens junctions, which are located at the end of myofibrils, gap junctions are often found in close association with mitochondria and and propagate electrical signals between cells by exchange of calcium and sodium ions. Whereas actin fibers of the contractile machinery are mainly composed of a-cardiac actin (red), actin filaments at gap junctions and the edges of Z- and intercalated discs, where the thin filaments are anchored, are composed of $\beta$-cytoplasmic actin (blue). (B-D) Proposed mechanism how Mena/NASP and all-Spectrin are thought to regulate cytoplasmic actin networks at Z-discs (B), intercalated discs (C), and gap junctions (D). For simplicity reasons, only Mena is depicted. Complexes of Mena/NASP and SH3i regulate $\beta$-actin networks by association at or near the barbed ends of $\beta$-actin filaments. Possibly, these complexes protect the barbed ends of thin filaments from capping by CapZ or modulate the geometry of the actin network by the intrinsic actin polymerization/bundling activity of Mena and VASP. Notably, heterotetrameric Spectrins, which are composed of two $\mathrm{a}$ - and two $\beta$-subunits, also regulate actin networks and are well known F-actin crosslinking proteins. Therefore disrupted Mena/VASP:SH3i complex formation in Mena ${ }^{\mathrm{GT} / G T} \mathrm{VASP}^{-/-}$mice may explain the disturbed morphology of Z- and intercalated discs, and the development of dilated cardiomyopathy and conduction abnormalities.

splice variant. In a complex with Cx43, ZO-1, and SH3i, Mena (and VASP) may regulate cytoplasmic networks and thereby stabilize the assembly and intercellular communication of gap junctions. In agreement with this hypothesis, blocking of actin dynamics by cytochalasin B treatment or blocking the association of $\mathrm{Cx} 43$ with the actin cytoskeleton led to a significant increase in the gap junction size $[39,40]$. Nevertheless, it remains unclear, why the enlarged gap junctions in our Mena ${ }^{\mathrm{GT} / \mathrm{GT}} \mathrm{VASP}^{-/-}$mice result in an impaired electrical conduction and prolonged PQ and QRS complexes 
in ECG recordings. Possibly, because of the lateralization of the gap junctions or a defective formation of gap junction channels between opposing cells. However, further experiments are required to address this question.

\section{Conclusions}

We propose a model how Mena/VASP and $\alpha I I-$ Spectrin regulate $\beta$-cytoplasmic actin networks at $\mathrm{Z}$ - and intercalated discs of the mammalian heart (Figure 13). Complexes of Mena/VASP and SH3i regulate $\beta$-actin networks by association at or near the barbed ends of $\beta$-actin filaments. Possibly, these complexes protect the barbed ends of thin filaments from capping or modulate the geometry of the actin network with implications for mechanical support, myofibrilogenesis, and ion channel function. Conversely, disrupted Mena/SH3i complex formation in Mena ${ }^{\mathrm{GT} / \mathrm{GT}} \mathrm{VASP}^{-/-}$ mice impairs the integrity of $\mathrm{Z}$ - and intercalated discs and results in dilated cardiomyopathy and conduction abnormalities.

\section{Methods \\ Generation of Mena ${ }^{\mathrm{GT} / \mathrm{GT}}$ and Mena ${ }^{\mathrm{GT} / \mathrm{GT}} \mathrm{VASP}^{-/-}$mice}

The mouse Mena gene is located on chromosome 1 spanning about $115 \mathrm{~kb}$ of genomic sequence (BLASTlike alignment tool, BLAT [41], Assembly NCBI37/mm9). To disrupt the Mena gene in mice, we generated a mouse strain, using the RRG138 embryonic stem (ES) cell line from BayGenomics (http://www.genetrap.org). In RRG138, the gene trap vector pGT0Lxf is inserted between exons 2 and 3 of the mouse Mena gene (Figure 2) and single insertion of the vector was confirmed by Southern blot analysis. 129/Ola ES cells heterozygous for the targeted mutation were microinjected into $\mathrm{C} 57 \mathrm{Bl} / 6$ blastocysts and implanted into pseudopregnant foster mothers. Male chimeras were mated with $\mathrm{C} 57 \mathrm{Bl} / 6$ females to obtain heterozygous $\mathrm{Mena}^{+/ \mathrm{GT}}$ mice. Offspring were genotyped using a forward primer located in exon 2 of the mouse Mena gene (me_F3: 5' - TGGGCAGAAAGATTCAA GACC - 3') either in combination with a reverse primer in Mena intron 2 (me_in2_R2: 5' - CCAGTTTCA ATGCCCATTCCTT - 3') to amplify the 1123 bp wild-type fragment or in combination with a reverse primer in the gene trap vector (pGT0Lxf_R2: 5' - CG GATCTCAAACTCTCСТCC - 3') to amplify the 824 bp knockout fragment. TA-cloning and sequencing of the knockout PCR product confirmed targeting of the mouse Mena gene and allowed to map the insertion of the gene trap vector 682 bp downstream of Mena exon 2 . VASP-deficient mice have been described previously [16] and were mated to Mena ${ }^{\mathrm{GT} / \mathrm{GT}}$ mice to generate Mena/ VASP double-deficient animals (Mena ${ }^{\mathrm{GT} / \mathrm{GT}} \mathrm{VASP}^{-/-}$). In the present study, 8-12 months old VASP-, Mena-, and Mena/
VASP double-deficient mice and controls of a mixed 129/ Ola X C57Bl/6 background were used for all experiments.

\section{Animal experiments}

All animal experiments were performed according to the relevant regulatory standards and were approved by the local government (license to $\mathrm{KS}$ and $\mathrm{PMB}$, \#621-2531.01-25/05, \#55.2-2531.01-62/08, and \#55.22531.01-95/11).

\section{Transverse aortic constriction (TAC)}

Male mice were anesthetized using isoflurane and the transversal aorta was prepared after jugular incision. A 27 -gauge needle was tied against the aorta using a 5-0 non-absorbable suture. After removal of the 27-gauge needle, skin was closed, and the mice were kept on a warming plate until recovery from anesthesia. After 3 weeks of TAC, mice were then sacrificed under anaesthesia, the hearts were weighed and the left ventricles were dissected for protein extraction.

\section{Echocardiography}

Serial transthoracic echocardiography (Aplio, Toshiba, Netherlands) was performed under light isoflurane anaesthesia and spontaneous respiration by a single researcher experienced in rodent echocardiography blinded to mouse genotype. From two-dimensional short axis imaging, endocardial borders were traced at endsystole and end-diastole utilizing a prototype off-line analysis system (NICE, Toshiba Medical Systems, The Netherlands). Measurements were performed at the mid-papillary muscle level. The end-systolic (smallest) and enddiastolic (largest) cavity areas were determined. Using the end-systolic (ESA) and-diastolic areas (EDA), fractional area changes were calculated ((EDA-ESA)/ EDA). Only animals with a heart rate greater than 450/ min were included.

\section{Invasive hemodynamics}

Terminal measurements of left ventricular pressure (LVP) were performed in $1 \%$ isoflurane anesthetized mice with a $1.4 \mathrm{~F}$ micromanometer-tipped catheter and MPVS Ultra amplifier (Millar Instruments). After 30 min of stabilization, $30 \mathrm{sec}$ of data were collected and analyzed with Chart 7.3 from ADInstruments running on PowerLab 16/30, including heart rate, systolic and diastolic LVP, and the first derivatives of LVP, $\pm \mathrm{dP} / \mathrm{dt}$. Only animals with a heart rate greater than $450 / \mathrm{min}$ were included.

\section{High resolution ECG recordings}

Mice were anesthetized using isoflurane (0.8\%) and kept on a warming plate $\left(37^{\circ} \mathrm{C}\right)$ throughout the experiment. Lead I ECG recordings were captured at a 
sampling rate of 4000 points per second (ADInstruments, PowerLab 8/35 with BIO amplifier) and original ECG recordings were analyzed with the ecg-auto software (version 2.5.0.3, emka technologies).

\section{Antibodies and fluorescent phalloidin}

Polyclonal rabbit anti-Mena antibodies were raised against a purified glutathione-S-transferase (GST, GE-Healthcare) fusion protein comprising mouse Mena amino acids L147-L279 (numbering according to NCBI protein ID AAC52863). Antibodies were immunoselected with a purified maltose-binding fusion protein (MBP, New England Biolabs) comprising the same mouse Mena residues. For double-immunofluorescence studies, we conjugated the purified Mena antibodies with fluorescein isothiocyanate (FITC, Sigma). Rabbit antibodies against the general $\alpha$ II-Spectrin protein isoform were generated and immunoselected with a purified GST fusion protein comprising amino acids E970D1025 of the human aII-Spectrin sequence (protein ID AAH53521). To remove $\alpha$ I-Spectrin- and GST-specific antibodies, the purified antiserum was immunodepleted with an excess of a GST fusion protein comprising $\alpha$ I-Spectrin residues A978-A1033 (protein ID NP_035595). Other antibodies used were anti-VASP (IG-731, Immunoglobe), anti-SH3i [30], anti-GST [10], anti-glyceraldehyde-3-phosphate dehydrogenase (GAPDH, mAB 374, Chemicon), anti- $\beta$-Tubulin (T8328, Sigma), anti- $\beta$-cytoplasmic actin (13E5, Cell Signalling; A1978, Sigma), anti- $\gamma$-cytoplasmic actin (A8481, Sigma), anti-CapZ (AB6017, Millipore), anti-Cx43 (sc-6560, Santa Cruz; 710700, Invitrogen), anti- $\alpha$-Actinin (A7811, Sigma). Horseradish peroxidase-conjugated secondary antibodies were from Dianova or eBioscience, fluorescence-conjugated antibodies (Alexa-fluor 488, 594, or 647) from Molecular Probes. Fluorescent phalloidin (P1951, Sigma; A22287, Invitrogen) was used to visualize F-actin.

\section{Organ lysates and Western blotting}

For Western blotting, $100 \mathrm{mg}$ mouse organ was homogenized in $2 \mathrm{ml}$ lysis buffer (2\% SDS, 1x PBS, 2x complete EDTA-free protease inhibitors (Roche)) using an Ultra Turrax. Following centrifugation for $30 \mathrm{~min}$ at 20,000 x g, protein content of the supernatant was determined by UV spectroscopy and $30 \mu \mathrm{g}$ total protein was resolved by polyacrylamide gel electrophoresis and transferred to nitrocellulose sheets. Membranes were blocked in 5\% skimmed milk in PBS, incubated with primary and horseradish peroxidase-conjugated secondary antibodies in blocking solution, and detection was performed with a chemiluminescence substrate (ECL Plus, GE-Healthcare). Equal protein loading was confirmed with $\beta$-Tubulin- or GAPDHspecific antibodies.

\section{Histological analyses}

For histological analysis, hearts were fixed in a neutral buffered methanol-free formalin solution for $24 \mathrm{~h}$ and then embedded in paraffin. $5 \mu \mathrm{m}$ transverse sections from the middle of wild-type and Mena/VASP doubledeficient hearts were stained with hematoxilin/eosin or $0.1 \%$ picrosirius red according to standard protocols. Representative photographs of the transilluminated stained sections on a Zeiss stage micrometer were collected with a Canon EOS 1000D digital camera.

For the analysis of cross sectional cell sizes, standard HE-stained heart sections were mounted with VectaShield mounting medium (Vector Laboratories), images were taken with a Keyence BZ digital microscope (Keyence, Germany), and cross-sectional areas of myocytes displaying round-shaped nuclei were quantified using the ImageJ software (NIH).

\section{RNA preparation and quantitative real-time PCR analysis}

RNA was extracted from frozen LV samples of 6 male mice per group using a Qiagen tissue RNA kit. cDNA was synthesized from $100 \mathrm{ng}$ of total RNA with Superscript (Invitrogen). Quantitative real-time PCR was performed (iCycler, Biorad) with commercially available TaqMan probes for ANP, $\beta$-MHC, SMA, Col I, Col III, and GAPDH (Invitrogen). Target gene mRNA levels in Mena ${ }^{\mathrm{GT} / \mathrm{GT}} \mathrm{VASP}^{-/-}$mice were normalized to GAPDH expressed relative to wild-type mice using the $2^{\wedge}(-\Delta \Delta \mathrm{CT})$ method.

\section{Isolation of cardiomyocytes}

Mice were anesthetized in a gas chamber with isoflurane, hearts were excised and mounted on a Langen dorff perfusion-system and were perfused with a nominally calcium-free Tyrodes' solution containing (in $\mathrm{mM}$ ) $\mathrm{NaCl} 113, \mathrm{KCl} 4.7, \mathrm{KH}_{2} \mathrm{PO}_{4}$ 0.6, $\mathrm{Na}_{2} \mathrm{HPO}_{4} \times 2 \mathrm{H}_{2} \mathrm{O}$ 0.6, $\mathrm{MgSO}_{4} \times 7 \mathrm{H}_{2} \mathrm{O}$ 1.2, $\mathrm{NaHCO}_{3} 12, \mathrm{KHCO}_{3}$ 10, HEPES 10, Taurine 30, BDM 10, glucose 5.5, phenol-red 0.032 for 4 min at $37^{\circ} \mathrm{C}$ and $\mathrm{pH}$ 7.4. Then, perfusion solution was switched to the same solution containing $7.5 \mathrm{mg} / \mathrm{ml}$ liberase 1 (Roche diagnostics, Mannheim, Germany), trypsin $0.6 \%$ and $0.125 \mathrm{mM} \mathrm{CaCl}_{2}$. Perfusion was continued for about $3 \mathrm{~min}$ until the heart became flaccid. Ventricular tissue was removed, cut into small pieces and dispersed until no solid cardiac tissue was left. Shortly after, cells were on plated Laminin-coated chamber slides (Nunc) and then prepared for immunofluorescence microscopy.

\section{GST pull-down and immunoprecipitation experiments} cDNA parts of the general mouse $\alpha \mathrm{II}$-Spectrin (protein ID CAM46235) or SH3i (AAI50942) isoform were subcloned into pGEX-6P2 vector (GE Healthcare) to generate constructs termed " $\mathrm{A}$ ", "SH3", " $\alpha 9$ ", and " $\alpha 9 \mathrm{~A}$ " 
(compare Figure 10A and B). Except for the alternatively spliced 20 amino acids TRITKEAGSVSLRMKQVEEL, which are only present in SH3i, the two protein isoforms are identical. A and $\alpha 9 \mathrm{~A}$ are specific for $\mathrm{SH} 3 i$ and encode amino acids T1053-L1072 and Q893-E1088, respectively. The W1004R mutant was obtained from $\alpha 9 \mathrm{~A}$ by replacing Trp1004 with an arginine residue by sitedirected mutagenesis. $\alpha 9$ is specific for the general $\alpha$ IISpectrin isoform and encodes amino acids Q893-E1068. GST+SH3 encodes the SH3 domain, which is present in both isoforms (E970-D1025). GST and the four GSTfusion proteins were expressed in E.coli and affinity purified on glutathione sepharose beads (compare Figure 10C). For GST pull-down assays, hearts of wildtype mice $(100 \mathrm{mg})$ were homogenized in ice-cold 40 mM Hepes- $\mathrm{NaOH}, \mathrm{pH}$ 7.4, $75 \mathrm{mM} \mathrm{NaCl}, 1 \% \mathrm{NP} 40$, and protease inhibitors (Roche) using a Polytron PT3100 homogenizer equipped with a PT-DA 3007 dispersing aggregate. Lysates were cleared by centrifugation $(17,000 \mathrm{x}$ $\mathrm{g}, 4^{\circ} \mathrm{C}, 15 \mathrm{~min}$ ) and incubated with $5 \mu \mathrm{g}$ immobilized GST or equimolar amounts of the GST-fusion proteins. Precipitated material was analyzed with anti-Mena and anti-VASP antibodies. GST pull-down assays with purified recombinant VASP were performed essentially as described [10].

For immunoprecipitation experiments, heart lysates were prepared as described above and then incubated with $5 \mu \mathrm{g}$ purified anti-Mena, anti- $\alpha$ II-Spectrin, or antiSH3i antibodies, followed by protein A-sepharose (GE Healthcare). Purified GST-specific antibodies served as control. After extensive washing in lysis buffer, precipitates were resuspended in SDS sample buffer, resolved by SDS-PAGE, and analyzed by Western blotting employing standard methods. To quantify the amount of Mena and Cx43, which is co-precipitated with the general $\alpha$ II-Spectrin or the SH3i variant, respectively, we performed densitometric scans (ImageJ version 1.45s, National Institute of Health, USA). For each IP/Western blot, the signal ratio of precipitated protein vs. a constant amount of heart lysate was calculated. For Mena and $\mathrm{Cx} 43$, ratios of $\mathrm{SH} 3 i$ precipitations are given relative to the ratios of $\alpha$ II-Spectrin precipitations.

\section{Immunohistochemistry}

All incubation and washing steps were performed at room temperature in a buffer containing $10 \mathrm{mM}$ PIPES pH6.8, $150 \mathrm{mM} \mathrm{NaCl}, 5 \mathrm{mM}$ EGTA, $5 \mathrm{mM}$ glucose, $5 \mathrm{mM} \mathrm{MgCl}$. $7 \mu \mathrm{m}$ mouse heart cryosections or isolated mouse cardiomyocytes were fixed in 4\% paraformaldehyde, extensively washed with $50 \mathrm{mM}$ ammonium chloride, permeabilized with $0.1 \%$ Triton, blocked in $10 \%$ goat serum for 1 $\mathrm{h}$, and overnight incubated with primary antibodies in $10 \%$ goat serum. Following extensive washing, samples were incubated with the fluorescence-conjugated secondary antibodies and fluorescence-conjugated phalloidin for 1.5 h. After additional three rounds of washing, samples were incubated with FITC-conjugated Mena antibodies, washed again, and mounted with Mowiol (Hoechst). Stained sections were investigated using a Leica SP5 confocal microscope equipped with a 100 -fold oil immersion objective. Images were acquired and prepared for presentation using the LAS AF 2.6.0 software from Leica. Pearson's correlation and Mander's overlap coefficients were calculated using the Intensity Correlation Analysis Plugin available for Image).

\section{Pre-embedding Immuno-electron microscopy}

Mouse cardiac tissue used for this study was fixed in $4 \%$ paraformaldehyde, $15 \%$ saturated picric acid in $0.1 \mathrm{M}$ phosphate buffer, $\mathrm{pH} 7.4$, overnight at $4^{\circ} \mathrm{C}$. Longitudinal sections were cut on a vibratome (Leica VT 1000S) at a thickness of $50 \mu \mathrm{m}$, blocked in 20\% NGS in PBS and were incubated with primary antibodies in phosphatebuffered saline containing 5\% normal goat serum (Vector Laboratories, Burlingame, CA, USA) overnight at $4^{\circ} \mathrm{C}$. After washes in PBS, the sections were incubated with $1.4 \mathrm{~nm}$ gold-coupled secondary antibodies in PBS (Nanoprobes, Stony Brook, NY, USA) overnight at $4^{\circ} \mathrm{C}$. After several washes the sections were postfixed in $1 \%$ glutaraldehyde in PBS for $10 \mathrm{~min}$ and then reacted with HQ Silver kit (Nanoprobes). After treatment with $\mathrm{OsO}_{4}$, sections were stained with uranyl acetate, dehydrated, and embedded in Durcupan resin (Fluka, Switzerland). Ultrathin sections were prepared (Ultracut S; Leica, Germany) and examined with a ZEISS 910 electron microscope.

\section{Abbreviations}

Cx43: Connexin 43; DCM: Dilated cardiomyopathy; dKO: MenaNASP doubledeficient; ES: Embryonic stem; EVH: Ena/NASP homology; GST: Glutathione-Stransferase; GT: Gene trap; IP: Immunoprecipitation; Mena: Mammalian enabled; M-KO: Mena-deficient; PKA: CAMP-dependent protein kinase; PKG: CGMP-dependent protein kinase; PRR: Proline-rich region; SH3: Src homology 3; SH3i: all-Spectrin splice variant; VASP: Vasodilator-stimulated phosphoprotein; V-KO: VASP-deficient.

\section{Competing interests}

The authors declare that they have no competing interests.

\section{Authors' contributions}

$\mathrm{PMB}, \mathrm{SF}$, and $\mathrm{KS}$ conceived and designed the study; PMB, CJM, KO, MA, BB, $H W, S G, S M F$, and AU performed the experiments; TF, JAU, IMA, SMF, TR, and SF contributed reagents and materials; PMB, CJM, MA, BB, SF, AU, MU, and KS analyzed the data; PMB and KS wrote the paper; SMF, TR, IF, WL, and SF critically reviewed the paper for important intellectual content. All authors read and approved the manuscript.

\section{Acknowledgements}

We thank Baygenomics for providing the RRG138 ES cell line. We are grateful to S. Umbenhauer for help with echocardiographic analyses, Dr. S. Tschernitz for help with histology, J. Jäpel and C. Thienel for support with cloning, Dr. C. Blume for helpful discussion, and D. Urlaub for technical support. We thank the group of Dr. Heinze for assistance in imaging and Prof. U. Walter for continuous support of the study. This work was supported in part by grants of the Deutsche Forschungsgemeinschaft (DFG) SFB 688 and the 
European Research Council grant (ERC-StG-2012-311575_F-12) to T. Renné, P. M. Benz, and S. Gambaryan and SFB 834 to P. M. Benz. S.M. Feller was supported by Cancer Research UK. K. Schuh was supported by grants from the Bundesministerium für Bildung und Forschung (BMBF01 EO1004) and the DFG (SCHU1600/2-1).

\section{Author details}

${ }^{1}$ Institute of Physiology I, University of Würzburg, D-97070 Würzburg, Germany. ${ }^{2}$ Institute for Vascular Signalling, Goethe-University Frankfurt, D-60590 Frankfurt, Germany. ${ }^{3}$ Department of Neurology, University of Bonn, D-53105 Bonn, Germany. ${ }^{4}$ Comprehensive Heart Failure Center, University of Würzburg, D-97070 Würzburg, Germany. Institute of Clinical Biochemistry and Pathobiochemistry, University of Würzburg, D-97070 Würzburg, Germany. ${ }^{6}$ Sechenov Institute of Evolutionary Physiology and Biochemistry, Russian Academy of Sciences, St. Petersburg, Russia. 'Department of Physiology, University of Maryland School of Medicine, Baltimore, MD 21201 USA. ${ }^{8}$ Institute of Human Genetics, University of Göttingen, D-37073 Göttingen, Germany. ${ }^{9}$ Department of Cardiovascular Physiology, Ruhr University Bochum, D-44780 Bochum, Germany. ${ }^{10}$ Biological Systems Architecture Group, University of Oxford, Oxford OX3 9DS UK. ${ }^{11}$ Section Tumor Biology, Institute of Molecular Medicine, ZAMED,

Martin-Luther-University Halle-Wittenberg, D-06120 Halle (Saale), Germany.

${ }^{12}$ Department of Molecular Medicine and Surgery and Center for Molecular Medicine, Karolinska Institutet, 17176 Stockholm, Sweden. ${ }^{13}$ Institute for Clinical Chemistry, University Hospital Eppendorf, D-20246 Hamburg, Germany.

Received: 16 January 2013 Accepted: 6 August 2013

Published: 12 August 2013

\section{References}

1. Perrin BJ, Ervasti JM: The actin gene family: function follows isoform. Cytoskeleton (Hoboken) 2010, 67:630-634.

2. Kee AJ, Gunning PW, Hardeman EC: Diverse roles of the actin cytoskeleton in striated muscle. J Muscle Res Cell Motil 2009, 30:187-197.

3. Liew CC, Dzau VJ: Molecular genetics and genomics of heart failure. Nat Rev Genet 2004, 5:811-825.

4. Bennett PM, Maggs AM, Baines AJ, Pinder JC: The transitional junction: a new functional subcellular domain at the intercalated disc. Mol Biol Cell 2006, 17:2091-2100.

5. Balasubramanian S, Mani SK, Kasiganesan H, Baicu CC, Kuppuswamy D: Hypertrophic stimulation increases beta-actin dynamics in adult feline cardiomyocytes. PLoS One 2010, 5:e11470.

6. Sechi AS, Wehland J: ENA/VASP proteins: multifunctional regulators of actin cytoskeleton dynamics. Front Biosci 2004, 9:1294-1310.

7. Gambaryan S, Hauser W, Kobsar A, Glazova M, Walter U: Distribution, cellular localization, and postnatal development of VASP and Mena expression in mouse tissues. Histochem Cell Biol 2001, 116:535-543.

8. Hansen SD, Mullins RD: VASP is a processive actin polymerase that requires monomeric actin for barbed end association. J Cell Biol 2010, 191:571-584.

9. Barzik M, Kotova TI, Higgs HN, Hazelwood L, Hanein D, Gertler FB, Schafer DA: Ena/VASP proteins enhance actin polymerization in the presence of barbed end capping proteins. J Biol Chem 2005, 280:28653-28662.

10. Benz PM, Blume C, Moebius J, Oschatz C, Schuh K, Sickmann A, Walter U, Feller SM, Renne T: Cytoskeleton assembly at endothelial cell-cell contacts is regulated by alphall-spectrin-VASP complexes. J Cell Biol 2008, 180:205-219.

11. Benz PM, Blume C, Seifert S, Wilhelm S, Waschke J, Schuh K, Gertler F, Munzel T, Renne T: Differential VASP phosphorylation controls remodeling of the actin cytoskeleton. J Cell Sci 2009, 122:3954-3965.

12. Scott JA, Shewan AM, den Elzen NR, Loureiro JJ, Gertler FB, Yap AS: Ena/VASP proteins can regulate distinct modes of actin organization at cadherin-adhesive contacts. Mol Biol Cell 2006, 17:1085-1095.

13. Kwiatkowski AV, Rubinson DA, Dent EW, van Veen EJ, Leslie JD, Zhang J, Mebane LM, Philippar U, Pinheiro EM, Burds AA, et al: Ena/VASP Is Required for neuritogenesis in the developing cortex. Neuron 2007, 56:441-455.

14. Lanier LM, Gates MA, Witke W, Menzies AS, Wehman AM, Macklis JD, Kwiatkowski D, Soriano P, Gertler FB: Mena is required for neurulation and commissure formation. Neuron 1999, 22:313-325.
15. Aszodi A, Pfeifer A, Ahmad M, Glauner M, Zhou XH, Ny L, Andersson KE, Kehrel B, Offermanns S, Fassler R: The vasodilator-stimulated phosphoprotein (VASP) is involved in CGMP- and CAMP-mediated inhibition of agonist-induced platelet aggregation, but is dispensable for smooth muscle function. Embo J 1999, 18:37-48.

16. Hauser W, Knobeloch KP, Eigenthaler M, Gambaryan S, Krenn V, Geiger J, Glazova M, Rohde E, Horak I, Walter U, Zimmer M: Megakaryocyte hyperplasia and enhanced agonist-induced platelet activation in vasodilator-stimulated phosphoprotein knockout mice. Proc Natl Acad Sci U S A 1999, 96:8120-8125.

17. Ahern-Djamali SM, Comer AR, Bachmann C, Kastenmeier AS, Reddy SK, Beckerle MC, Walter U, Hoffmann FM: Mutations in Drosophila enabled and rescue by human vasodilator-stimulated phosphoprotein (VASP) indicate important functional roles for Ena/VASP homology domain 1 (EVH1) and EVH2 domains. Mol Biol Cell 1998, 9:2157-2171.

18. Menzies AS, Aszodi A, Williams SE, Pfeifer A, Wehman AM, Goh KL, Mason CA, Fassler R, Gertler FB: Mena and vasodilator-stimulated phosphoprotein are required for multiple actin-dependent processes that shape the vertebrate nervous system. J Neurosci 2004, 24:8029-8038.

19. Baines AJ, Pinder JC: The spectrin-associated cytoskeleton in mammalian heart. Front Biosci 2005, 10:3020-3033.

20. Bennett $V$, Healy J: Membrane domains based on ankyrin and spectrin associated with cell-cell interactions. Cold Spring Harb Perspect Biol 2009, 1:a003012.

21. Bennett V, Baines AJ: Spectrin and ankyrin-based pathways: metazoan inventions for integrating cells into tissues. Physiol Rev 2001, 81:1353-1392.

22. Bennett PM, Baines AJ, Lecomte MC, Maggs AM, Pinder JC: Not just a plasma membrane protein: in cardiac muscle cells alpha-Il spectrin also shows a close association with myofibrils. J Muscle Res Cell Motil 2004, 25:119-126.

23. Stankewich MC, Cianci CD, Stabach PR, Ji L, Nath A, Morrow JS: Cell organization, growth, and neural and cardiac development require alphall-spectrin. J Cell Sci 2011, 124:3956-3966.

24. Suzuki J, Fukuda M, Kawata S, Maruoka M, Kubo Y, Takeya T, Shishido T: A rapid protein expression and purification system using Chinese hamster ovary cells expressing retrovirus receptor. J Biotechnol 2006, 126:463-474.

25. Gertler FB, Niebuhr K, Reinhard M, Wehland J, Soriano P: Mena, a relative of VASP and Drosophila Enabled, is implicated in the control of microfilament dynamics. Cell 1996, 87:227-239.

26. Ullrich M, Schuh K: Gene trap: knockout on the fast lane. Methods Mol Biol 2009, 561:145-159.

27. Wiebe MS, Nichols RJ, Molitor TP, Lindgren JK, Traktman P: Mice deficient in the serine/threonine protein kinase VRK1 are infertile due to a progressive loss of spermatogonia. Biol Reprod 2010, 82:182-193.

28. Voss AK, Thomas T, Gruss P: Efficiency assessment of the gene trap approach. Dev Dyn 1998, 212:171-180.

29. Heineke J, Molkentin JD: Regulation of cardiac hypertrophy by intracellular signalling pathways. Nat Rev Mol Cell Biol 2006, 7:589-600.

30. Ursitti JA, Petrich BG, Lee PC, Resneck WG, Ye X, Yang J, Randall WR, Bloch $R J$, Wang Y: Role of an alternatively spliced form of alphall-spectrin in localization of connexin 43 in cardiomyocytes and regulation by stress-activated protein kinase. J Mol Cell Cardiol 2007, 42:572-581.

31. Li Q, Lau A, Morris TJ, Guo L, Fordyce CB, Stanley EF: A syntaxin 1, Galpha (o), and $\mathrm{N}$-type calcium channel complex at a presynaptic nerve terminal: analysis by quantitative immunocolocalization. J Neurosci 2004, 24:4070-4081.

32. Saksela K, Permi P: SH3 domain ligand binding: What's the consensus and where's the specificity? FEBS Lett 2012, 586:2609-2614.

33. Sartoretto JL, Jin BY, Bauer M, Gertler FB, Liao R, Michel T: Regulation of VASP phosphorylation in cardiac myocytes: differential regulation by cyclic nucleotides and modulation of protein expression in diabetic and hypertrophic heart. Am J Physiol Heart Circ Physiol 2009, 297:H1697-H1710.

34. Aguilar F, Belmonte SL, Ram R, Noujaim SF, Dunaevsky O, Protack TL, Jalife J, Massey TH, Gertler FB, Blaxall BC: Mammalian enabled (Mena) is a critical regulator of cardiac function. Am J Physiol Heart Circ Physiol 2011, 300:H1841-H1852.

35. Eigenthaler M, Engelhardt S, Schinke B, Kobsar A, Schmitteckert E, Gambaryan S, Engelhardt CM, Krenn V, Eliava M, Jarchau T, et al: Disruption of cardiac Ena-VASP protein localization in intercalated disks causes dilated cardiomyopathy. Am J Physiol Heart Circ Physiol 2003, 285:H2471-H2481. 
36. Bear JE, Gertler FB: Ena/VASP: towards resolving a pointed controversy at the barbed end. J Cell Sci 2009, 122:1947-1953.

37. Larsson $H$, Lindberg $U$ : The effect of divalent cations on the interaction between calf spleen profilin and different actins. Biochim Biophys Acta 1988, 953:95-105.

38. Dbouk HA, Mroue RM, El-Sabban ME, Talhouk RS: Connexins: a myriad of functions extending beyond assembly of gap junction channels. Cell Commun Signal 2009, 7:4.

39. Rassat J, Robenek H, Themann H: Cytochalasin B affects the gap and tight junctions of mouse hepatocytes in vivo. J Submicrosc Cytol 1982, 14:427-439.

40. Hunter AW, Barker RJ, Zhu C, Gourdie RG: Zonula occludens-1 alters connexin43 gap junction size and organization by influencing channel accretion. Mol Biol Cell 2005, 16:5686-5698.

41. Kent WJ: BLAT-the BLAST-like alignment tool. Genome Res 2002, 12:656-664.

doi:10.1186/1478-811X-11-56

Cite this article as: Benz et al: Mena/VASP and all-Spectrin complexes regulate cytoplasmic actin networks in cardiomyocytes and protect from conduction abnormalities and dilated cardiomyopathy. Cell

Communication and Signaling 2013 11:56.

\section{Submit your next manuscript to BioMed Central and take full advantage of:}

- Convenient online submission

- Thorough peer review

- No space constraints or color figure charges

- Immediate publication on acceptance

- Inclusion in PubMed, CAS, Scopus and Google Scholar

- Research which is freely available for redistribution 